\title{
Performance Analysis and Monitoring of VARIOUS ADVANCED DIGITAL MODULATION AND MUlTiPleXiNg TECHNIQUES OF F.O.C WiTHIN AND BEYOND $400 \mathrm{~GB} / \mathrm{s}$.
}

\author{
Sumant Ku. Mohapatra, Ramya Ranjan Choudhury, Rabindra Bhojray and \\ Pravanjan Das \\ Department of Electronics \& Telecommunication Engineering, \\ Trident Academy of Technology, B.P.U.T, Bhubaneswar, Odisha, India
}

\begin{abstract}
To achieve better calculative performance in optical fiber communication and for simplicity of implementation different digital modulation, detection and multiplexing techniques are used. These techniques maximize the spectral efficiency. This paper reviews a tabular comparative analysis with 3D graphical representation for different optical digital modulation formats and multiplexing techniques within and beyond $400 \mathrm{~Gb} / \mathrm{s}$. In this particular article we survey about different parameters related to digital fiber optic communication.
\end{abstract}

\section{KEYWORDS}

OFDM, Digital Modulation formats, Multiplexing techniques, QAM \& WDM.

\section{INTRODUCTION}

Now a days by digital communication one can improve the performance of OSNR sensitivity, Bit error rate, nominal range, sensitivity to non-linear distortion, transmission, attenuation profile, modulated bandwidth efficiency, information capacity, Spectral efficiency etc, The goal behind each type of optical modulation and multiplexing techniques is to increase the data rate, transmission fidelity and transmission distance between stations. Over the last years several types of modulation techniques are designed which consists of 2.5, 10,20, 25, 40 and $100 \mathrm{~Gb} / \mathrm{s}$ wavelength channels. But now a day the data rate with respect to the channel increases to 400 $\mathrm{Gb} / \mathrm{s}$ and above. Media Access control parameters, physical layers, and management parameter [17] using 4-channels with 25Gb/s. $107 \mathrm{~Gb} / \mathrm{s} \mathrm{NRZ-DQPSK} \mathrm{transmission} \mathrm{at} 1.0 \mathrm{~b} / \mathrm{s} / \mathrm{Hz}$ over 12 $100 \mathrm{Km}$ have been introduced [18] by P.J.Winzer including 6 optical routing nodes (published in Proc.OFC2007, post deadline paper PDP24). Now a days in modern digital optical fiber communication to improve transmission data rate200Gb/s , 400Gb/s , $800 \mathrm{~Gb} / \mathrm{s}, 1000 \mathrm{~Gb} / \mathrm{s}$, 1Tbit/s and above have been used. This paper also provides a tabular manner survey of modulation methods, with emphasis on probability of error, photons per pulse and spectral efficiency and other DFOC parameters. Multiplexing is a promising technique in optical fiber communication. Different types of fiber optic multiplexing techniques such as OTDM, OFDM, COFDM, WDM, CWDM and DWDM are analyzed in a tabular manner compared with different digital modulation formats. The comparative analysis taking suitability of various modulation formats over bit error rates of 0.004 is studied in this approach. The robustness of DPSK direct detection transmission format in standard fiber WDM systems have been published in2000 [33]. 
International Journal of Computer Networks \& Communications (IJCNC) Vol.6, No.2, March 2014

In this paper we mainly emphasize on WDM, CWDM and DWDM. W. Idler publishes WDM field over $764 \mathrm{Km}$ SSMF with 16-112Gb/s NRZ DQPSK[34]. These performances are comparatively analyzed in a tabular manner and also by different 3D graphical formats.

\section{Classification of Digital Optical Fiber Modulation and MULTIPLEXING TECHNIQUES.}

\begin{tabular}{|c|c|c|c|}
\hline Sl. No. & DFOC Format & Type & Notation \\
\hline \multirow{18}{*}{01.} & \multirow{18}{*}{$\begin{array}{l}\text { Digital } \\
\text { modulation } \\
\text { formats }\end{array}$} & $\begin{array}{l}\text { On-OFF keying /Binary } \\
\text { Amplitude }\end{array}$ & OOK/BASK \\
\hline & & Binary frequency shift keying & BFSK \\
\hline & & Binary Phase Shift Keying & BPSK \\
\hline & & Differential Phase Shift Keying & DPSK \\
\hline & & Return to zero DPSK & RZ-DPSK \\
\hline & & Quadrature Phase Shift Keying & QPSK \\
\hline & & Differential QPSK & DQPSK \\
\hline & & Return to zero DQPSK & RZ-DQPSK \\
\hline & & Return to zero DPSK-3ASK & RZ-DPSK-3ASK \\
\hline & & $\begin{array}{l}\text { Polarization division multiplexing } \\
\text { QPSK }\end{array}$ & PM-QPSK/DP-QPSK \\
\hline & & $\begin{array}{l}\text { PM-Orthogonal frequency } \\
\text { division }\end{array}$ & $\begin{array}{l}\text { PM-OFDM-QPSK/DP- } \\
\text { OFDM-QPSK }\end{array}$ \\
\hline & & $\begin{array}{lll}\text { Optical } & \text { Polarization } & \text { FDM-RZ- } \\
\text { DQPSK } & & \\
\end{array}$ & OP-FDM-RZ-DQPSK \\
\hline & & $\begin{array}{l}\text { Polarization division multiplexing } \\
\text { DQPSK }\end{array}$ & PM-DQPSK or DP-DQPSK \\
\hline & & $\begin{array}{l}\text { M-ary Quadrature amplitude } \\
\text { modulation }\end{array}$ & M-QAM \\
\hline & & Minimum Shift Keying & MSK \\
\hline & & Gaussian MSK & GMSK \\
\hline & & Single Carrier Modulation formats & SCM \\
\hline & & Multicarrier Modulation formats & MCM \\
\hline \multirow{9}{*}{02.} & \multirow{9}{*}{$\begin{array}{l}\text { Digital } \\
\text { Multiplexing } \\
\text { Formats }\end{array}$} & Optical Time Division Multiplexing & OTDM \\
\hline & & Subcarrier Multiplexing & SCM \\
\hline & & $\begin{array}{l}\text { Orthogonal Frequency Division } \\
\text { Multiplexing }\end{array}$ & OFDM(UNCODED) \\
\hline & & $\begin{array}{ll}\text { Coded Frequency } & \text { Division } \\
\text { Multiplexing } & \\
\end{array}$ & COFDM(coded) \\
\hline & & Duty Cycle Division Multiplexing & DCDM \\
\hline & & $\begin{array}{l}\text { Optical Polarization } \\
\text { Multiplexing }\end{array}$ & OPDM \\
\hline & & Wavelength Division Multiplexing & WDM \\
\hline & & Coarse WDM & CWDM \\
\hline & & Dense WDM & DWDM \\
\hline
\end{tabular}


International Journal of Computer Networks \& Communications (IJCNC) Vol.6, No.2, March 2014

\section{Optical Fiber Digital Modulation Formats.}

Modulation is a technique by which the digital information is printed onto an optical carrier [16] and in its most general sense also including coding to present transmission errors. In digital optical fibers the electromagnetic waves with frequencies of nearly $200 \mathrm{THz}$ are used to transfer information from one point to another.

\subsection{On-Off Keying/Binary Amplitude Shift Keying}

In BFSK, for better demodulation performance matched filter detectors are used. The information capacity is better than BASK indicated in Table-5. It is not efficient due to its hardware design of receiver, is complex as directed in Table-2.

\subsection{Binary Phase Shift Keying}

In BFSK, for better demodulation performance matched filter detectors are used. The information capacity is better than BASK indicated in Table-5. It is not efficient due to its hardware design of receiver, is complex as directed in Table-2.

\subsection{Binary Phase Shift Keying}

In BPSK error performance is very less as compared to BASK and BFSK. It is widely used for satellite communication. The binary 1 is signed as sin $\omega$ t and 0 signed as $-\sin \omega \mathrm{t} .2,4,8,16$ BPSK formats using coherent detection techniques to improve their BER performance as shown in Table-4.The information capacity of BPSK is twice times the BFSK indicated in Table-5.

\subsection{Differential Phase Shift Keying}

The non-linear propagation [33] in Optical Transmission systems is only valid for DPSK FOC digital modulation techniques. For $400 \mathrm{~Gb} / \mathrm{s}$ performance, it requires DPSK receiver Optical channel monitoring in optical line system. The maximum bandwidth is $80 \mathrm{ps} / \mathrm{nm}$ for RZ-DPSK shown in Table-10.

\subsection{Non Return to Zero /Return to Zero Differential Phase Shift Keying}

In NRZ/RZ-DPSK, the receiver design consists of one interferometric detector and two photo detectors which increases the hardware complexity in comparison to transmitter design which uses only one modulator at $400 \mathrm{~Gb} / \mathrm{s}$ aggregation bit rate shown in Table-10.

\subsection{Quadrature Phase Shift Keying}

In QPSK, the bandwidth efficiency is very high in comparison to other primary optical digital modulation techniques as illustrated in Table-5. Also the information capacity is twice the Binary Frequency Shift Keying which gives major effect on different primary modulation techniques.

\subsection{Differential Quadrature Phase Shift Keying}

At $400 \mathrm{~Gb} / \mathrm{s}$ DQPSK requires two modulators which improves the performance in comparison to QPSK. Also the BER graph of DQPSK gives better results when probability of error is taken into account. This four level version of DPSK has the advantage of tolerating better dispersion which is narrated in Table-7. 
International Journal of Computer Networks \& Communications (IJCNC) Vol.6, No.2, March 2014

\subsection{Return to Zero - Differential Quadrature Phase Shift Keying}

To get RZ-DQPSK signal, two phase modulators are cascaded for the modulation of the optical phase by 0 to $\pi / 2$ and 0 to $\pi / 4$ applying binary modulation. The Optical signal-to-noise-ratio tolerance is higher than DQPSK that results in better performance in the context of signal quality at $111 \mathrm{~Gb} / \mathrm{s}$ [35] and at $112 \mathrm{~Gb} / \mathrm{s}$ OUT-4 channel bit-rate [34], [36].The maximum bandwidth (ps/nm) of this format is half that of NRZ-DQPSK as compared in Table-10.

\subsection{Return to Zero - Differential Phase Shift Keying - 3 Amplitude Shift Keying}

This is a very fundamental mixer of ASK modulation and phase modulation. In RZ-DPSK-3ASK modulation formats 2.5 bits are coded in one symbol which leads to symbol rate of 43 Gbauds [3738], [65- 66] for support of the OUT-4 line-rate [67] of $112 \mathrm{~Gb} / \mathrm{s}$. This modulation technique when applied to field fiber has OSNR limitation, but this could be improved by reducing channel bit-rate.

\subsection{Polarization Mode -QPSK/Differential Phase -QPSK}

The $100 \mathrm{~Gb} / \mathrm{s}$ PM-QPSK transmission process [16] running at a symbol rate of $25-28 \mathrm{Gbaud}$ is widely applied with offline signal processing of electrical signal which is measured by 4- channel high speed real time Oscilloscopes acting as fast A/D converters[28-29], [40], [69]. Table-4 shows that the PM-QPSK format has higher modulation efficiency compared to QPSK format.

\subsection{Polarization Mode OFDM-QPSK/ Differential Phase -OFDM-QPSK}

Another commercially available $100 \mathrm{~Gb} / \mathrm{s}$ transponder applies two narrow spaced (20GHz) optical carries each modulated with PM-QPSK formats based on 14 Gbaud modulation [41],[16]. The hardware implementation features of transmitter and receiver of this modulation technique is given in Table-10. It has highest estimated reach of about $2000 \mathrm{Km}$ rather than QPSK, DQPSK and PM-DQPSK as suggested in Table-7.

\subsection{Optical Polarization -FDM-RZ-DQPSK}

To carry two optical carrier there are polarizations can be used to eliminate the fast automatic optical polarization de-multiplexers[16]. In this modulation format two carriers are alternatively multiplexed and de-multiplexed with optical fiber at 28 Gbaud. The compatibility with $100 \mathrm{~Gb} / \mathrm{s}$ $\& 400 \mathrm{~Gb} / \mathrm{s}$ is being positive w.r.t PM-OFDM-QPSK as shown in Table-7.

\subsection{Polarization Mode -DQPSK / Differential Phase -DQPSK}

By applying polarization division multiplexing (PM), we can reduce the symbol rate. As a result the line-rate doubles or the symbol rate becomes half [16]. The 28Gbaud modulation formats supports the 400G DWDM transmission with $50 \mathrm{GHz}$ channel spacing. Table-7 indicates that the OSNR tolerance (dB) @ BER 4X10-3 is higher than OP-FDM-RZ-DQPSK but less than RZDPSK-3ASK format.

\subsection{M-QAM}

'M' number of binary bits are transmitted in a particular slot in this modulation scheme [16]. This technique currently is of high research interest and is illustrated at submarine transmission configurations[70] using RZ at PM-QPSK. Polarization multiplexed 16- QAM signals have been 
International Journal of Computer Networks \& Communications (IJCNC) Vol.6, No.2, March 2014

realized by multi-level generation using passive combination of binary signals to achieve 224 $\mathrm{Gb} / \mathrm{s}$ channel rate (200G + FEC overhead)[71-73] and for higher than $400 \mathrm{~Gb} / \mathrm{s}$ channel rate [74]. Using Polarization multiplexing and QAM modulation format transmission lengths between $670 \mathrm{~km}$ to $1500 \mathrm{~km}$ have been demonstrated [71-73]. RF-assisted optical Dual carrier $112 \mathrm{~Gb} / \mathrm{s}$ polarization multiplexed 16-QAM is applied to achieve $112 \mathrm{~Gb} / \mathrm{s}$ channel rate[75]. According to Table-8, we conclude a comparative analysis between different M-QAM modulation techniques having different bit rates $(\mathrm{Gb} / \mathrm{s})$. A channel rate of $400 \mathrm{~Gb} / \mathrm{s}$ has been achieved using 16-QAM recently with polarization multiplexing.

\subsection{Minimum Shift Keying}

The new optical minimum shift keying modulation schemes have the high spectral efficiency as compared to other digital modulation formats. The transmitters for optical MSK based on two MZM similar to the transmitter for DQPSK. As compared to other modulation formats the spectrum is not compact enough to realize data rates as shown in Table-2.

\subsection{Gaussian Minimum Shift Keying}

GMSK is a digital optical binary modulation schemes and is treated as a extension of optical Minimum Shift Keying technique. In this format the side lobe levels of the spectrum are again minimized by passing the modulating NRZ data waveform through a pre-modulation Gaussian pulse-shaping filter. It promotes ISI at higher bit rate transmission than MSK as compared in Table-2.

\subsection{Sub-Carrier Modulation}

In this format $2 \mathrm{xm}$ bits are transmitted per symbol. Various constellations [16],[42] can be applied for PM-QAM modulation format. To optimize the signal error with M-QAM constellation by Nyquist filtering towards Nyquist wavelength division multiplexing which is currently of high research interest which has been demonstrated at submarine transmission configurations[70] using RZ at PM-QPSK. Table-8 gives an overview in single channel M-QAM options from $200 \mathrm{~Gb} / \mathrm{s}$ to $1 \mathrm{~Tb} / \mathrm{s}$.

\subsection{Multi-Carrier Modulation}

Multi-carrier modulation format approach supports high bandwidth channels [76]. Forming inverse fourier transform, Signal Processing is applied in the transmitter. As OFDM has rectangular shape, high capacity transmission can be performed by close allocation of multiple OFDM signals in the frequency domain without guard bands. The orthogonal multiplexing behavior of PM-QPSK modulation has been depicted in Table-7. A number of transmission experiments using polarization multiplexed O-OFDM and PM-O-OFDM have been reported [16], [77], [44-45] transporting Tb/s super channels over submarine distances [78].

\section{OPTICAL FibER Digital MULTIPLEXING Formats}

Multiple users can transmit data simultaneously through a single optical fiber link by digital multiplexing techniques described in this section. This is widely employed in optical communication systems due to its capability to increase the channel utilization and decrease system costs. 
International Journal of Computer Networks \& Communications (IJCNC) Vol.6, No.2, March 2014

\subsection{Optical Time Division Multiplexing}

In OTDM the bit-rate of digital optical fiber systems is increased beyond the bandwidth capabilities of the opto-electronics. [59-60].

\subsection{Sub-Carrier Multiplexing}

The subcarrier enables multiple broadband signals to be transmitted over single mode fiber and appear particularly attractive for video distribution systems. Also with SCM, the orthodox microwave solid-state devices could be used to further divide the intensity modulation available, thereby increasing the bandwidth.

\subsection{Orthogonal Frequency Division Multiplexing}

Reduction in the channel spacing is a major adaptability, which is employed in the orthogonal set of signals and is known as Orthogonal Frequency Division Multiplexing [76-78].Table-7 shows an comparative analysis of OFDM with different modulation formats.

\subsection{Coded Frequency Division Multiplexing}

The Coded Frequency Division Multiplexing is also called as OFDM [77], is a system where individual data bits of a word are coded onto individual carriers. Mutually orthogonal frequency carriers are used over one symbol period in this method. It has higher spectral efficiency OPFDM-RZ-DQPSK as shown in Table-7.

\subsection{Duty Cycle Division Multiplexing}

In this Duty Cycle Division Multiplexing (DCDM) technique [75], different users sign with different RZ duty cycles and the combine together synchronously to form a multi-level step shape signal.

\subsection{Optical Polarization Division Multiplexing}

Optical Polarization Division Multiplexing is a technique in which the capacity of the system and spectral efficiency is enhanced by using two independently modulated channels keeping the wavelength constant [75]. A brief comparison between 4-QAM(4 bits/symbol), 8-QAM (6 bits/symbol) and 16-QAM(8 bits/symbol) on the basis of polarization multiplexed transmission is illustrated in Table-12.

\subsection{Wavelength Division Multiplexing}

WDM is an optical modulation technique in optical fiber communication employing more than one wavelength. In this communication format, multiple optical carrier signals on a single fiber optic cable is multiplexed by using different wavelengths of laser light to carry various signals . In multimode the $850 \mathrm{~nm}, 1310 \mathrm{~nm}$ wavelengths are used [34-36]. In single mode 1310 and 1550 $\mathrm{nm}$ are used[43].The OSNR (dBm), maximum bandwidth (ps/nm), CRF (GHz) like parameters are clearly compared in Table-10. 
International Journal of Computer Networks \& Communications (IJCNC) Vol.6, No.2, March 2014

\subsection{Coarse WDM}

Coarse WDM gives the ability to combine upto 18 wavelengths onto one fiber. The spacing of these eighteen wavelengths which are employed evenly from 1270-1610 nm in $20 \mathrm{~nm}$ increments have been discussed.. The aggregate fiber capacity of CWDM is only $20-40 \mathrm{~Gb} / \mathrm{s}(70 \mathrm{Km})$ as indicated in Table-14.

\subsection{Dense WDM}

Dense WDM takes bandwidth and throughput to higher level. DWDM permits up to 80 wavelengths [46] to share are fiber[32]. The aggregate fiber capacity of DWDM is higher than CWDM that is up to $1 \mathrm{~Tb} / \mathrm{s}(900 \mathrm{Km})$ as indicated in figure 15 of Table-18.

Table 1. Comparative analysis of different PSK Schemes

\begin{tabular}{|l|l|l|l|c|}
\hline $\begin{array}{l}\text { Digital PSK } \\
\text { Modulation } \\
\text { Techniques }\end{array}$ & Probability of Error & Degradation & $\begin{array}{l}\text { Power Spectral Density } \\
\text { (PSD) }\end{array}$ & $\boldsymbol{B}_{\text {Null }}$ \\
\hline BPSK & $Q\left(\sqrt{\frac{2 E_{b}}{N_{0}}}\right)$ & $0 \mathrm{~dB}($ ref. $)$ & $\mathrm{A}^{2} \mathrm{~T}_{\mathrm{b}}\left(\frac{\operatorname{Sin} \pi f T_{b}}{\pi f T_{b}}\right)^{2}$ & $\frac{2}{T_{b}}$ \\
\hline DEBPSK & $\approx 2 Q\left(\sqrt{\frac{2 E_{b}}{N_{0}}}\right)$ & $<0.5 \mathrm{~dB}$ & $\mathrm{~A}^{2} \mathrm{~T}_{\mathrm{b}}\left(\frac{\operatorname{Sin} \pi f T_{b}}{\pi f T_{b}}\right)^{2}$ & $\frac{2}{T_{b}}$ \\
\hline $\begin{array}{l}\text { DBPSK } \\
\text { (Optimum) }\end{array}$ & $\frac{1}{2} e^{-E_{b} / N_{0}}$ & $0.5-1 \mathrm{~dB}$ & $\mathrm{~A}^{2} \mathrm{~T}_{\mathrm{b}}\left(\frac{\operatorname{Sin} \pi f T_{b}}{\pi f T_{b}}\right)^{2}$ & $\frac{2}{T_{b}}$ \\
\hline $\begin{array}{l}\text { QPSK } \\
\text { DEQPSK }\end{array}$ & $Q\left(\sqrt{\frac{2 E_{b}}{N_{0}}}\right)$ & $0 \mathrm{~dB}(\mathrm{ref})$. & $2 \mathrm{~A}^{2} \mathrm{~T}_{\mathrm{b}}\left(\frac{\operatorname{Sin} 2 \pi f T_{b}}{2 \pi f T_{b}}\right)^{2}$ & $\frac{1}{\mathrm{~T}_{\mathrm{b}}}$ \\
\hline $\begin{array}{l}\text { DQPSK } \\
\text { (Optimum) }\end{array}$ & $\approx Q\left(\sqrt{\frac{2 E_{b}}{N_{0}}}\right)$ & $<0.5 \mathrm{~dB}$ & $2 \mathrm{~A}^{2} \mathrm{~T}_{\mathrm{b}}\left(\frac{\operatorname{Sin} 2 \pi f T_{b}}{2 \pi f T_{b}}\right)^{2}$ & $\frac{1}{\mathrm{~T}_{\mathrm{b}}}$ \\
\hline $\begin{array}{l}\text { DMPSK } \\
\text { (Optimum) }\end{array}$ & $\approx \frac{\pi}{4 \sqrt{2}} Q\left(\sqrt{\frac{2 n E_{b}}{N_{0}}} \sin \frac{\pi}{\sqrt{2} M}\right)$ & $2-3 \mathrm{~dB}$ & $2 \mathrm{~A}^{2} \mathrm{~T}_{\mathrm{b}}\left(\frac{\operatorname{Sin} 2 \pi f T_{b}}{2 \pi f T_{b}}\right)^{2}$ & $\frac{1}{\mathrm{~T}_{\mathrm{b}}}$ \\
\hline
\end{tabular}


International Journal of Computer Networks \& Communications (IJCNC) Vol.6, No.2, March 2014

Table 2. Modulation parameters of different Digital modulation techniques in $40 \mathrm{~Gb} / \mathrm{s}$ modulation formats.

\begin{tabular}{|c|c|c|c|c|}
\hline $\begin{array}{l}\text { Digital } \\
\text { Modulation }\end{array}$ & $\begin{array}{l}\text { Demodulation } \\
\text { performance }\end{array}$ & $\begin{array}{l}\text { Error } \\
\text { performance }\end{array}$ & Advantages & Disadvantages \\
\hline BASK & $\begin{array}{l}\text { Easy } \\
\text { demodulation }\end{array}$ & $\begin{array}{l}\text { Restricted in } \\
\text { linear region }\end{array}$ & $\begin{array}{l}\text { Hardware } \\
\text { Implementations } \\
\text { simple and low }\end{array}$ & Poor BW \\
\hline BFSK & $\begin{array}{l}\text { Matched filter } \\
\text { detectors used }\end{array}$ & $\begin{array}{l}\text { Performs } \\
\text { well }\end{array}$ & Same as Bask & $\begin{array}{l}\text { Complex } \\
\text { Hardware design }\end{array}$ \\
\hline BPSK & $\begin{array}{l}\text { Receiver circuit } \\
\text { is complex. }\end{array}$ & $\begin{array}{l}\text { Small error } \\
\text { rate }\end{array}$ & $\begin{array}{l}\text { Used only for } \\
\text { satellite }\end{array}$ & Inefficient \\
\hline DPSK & $\begin{array}{l}\text { Receiver } \\
\text { requires } \\
\text { memory }\end{array}$ & $\begin{array}{l}\text { Required } 3 \\
\text { dB less than } \\
\text { BFSK }\end{array}$ & $\begin{array}{l}\text { Introduces the } \\
\text { complexities } \\
\text { of receiver }\end{array}$ & $\begin{array}{l}\text { Efficient less } \\
\text { than coherent } \\
\text { PSK }\end{array}$ \\
\hline QPSK & $\begin{array}{l}\text { Phase shift } \\
\text { detection is used }\end{array}$ & $\begin{array}{l}\text { Better over } \\
\text { BPSK and } \\
\text { BFSK }\end{array}$ & $\begin{array}{l}\text { Bandwidth } \\
\text { efficient } \\
\text { than BPSK }\end{array}$ & $\begin{array}{l}\text { Hardware } \\
\text { design of } \\
\text { receiver is }\end{array}$ \\
\hline 64 QAM & $\begin{array}{l}\text { Coherent } \\
\text { detection }\end{array}$ & $\begin{array}{l}\text { Same as } \\
\text { OAM }\end{array}$ & $\begin{array}{l}\text { Very efficient } \\
\text { spectral efficiency }\end{array}$ & $\begin{array}{l}\text { BW is same as } \\
\text { ASK and PSK }\end{array}$ \\
\hline GMSK & $\begin{array}{l}\text { Bandwidth time } \\
\text { product is } \\
\text { measured by } \\
\text { SNR Vs BER }\end{array}$ & $\begin{array}{l}\text { The carrier lags } \\
\text { or leads by } 90^{0} \\
\text { over bit period } \\
\text { w.r.t BT. }\end{array}$ & $\begin{array}{l}\text { Constant envelope, } \\
\text { spectrally efficient }\end{array}$ & $\begin{array}{l}\text { It promotes ISI at } \\
\text { higher bit rate } \\
\text { transmission }\end{array}$ \\
\hline
\end{tabular}

Table 3. Comparison of performance and implementation for $400 \mathrm{~Gb} / \mathrm{s}$.

\begin{tabular}{|c|c|c|}
\hline $\begin{array}{l}\text { Digital } \\
\text { Modulatio } \\
\text { n } \\
\text { Technique } \\
\text { S }\end{array}$ & $\begin{array}{l}400 \mathrm{~Gb} / \mathrm{s} \text { performance and } \\
\text { implementation Advantages }\end{array}$ & $\begin{array}{l}400 \mathrm{~Gb} / \mathrm{s} \text { performance and } \\
\text { implementation Disadvantages }\end{array}$ \\
\hline NRZ & - "baseline" (no OSNR penalty) & $\begin{array}{l}\text { - } \quad \text { "baseline" : Single modulator stage. } \\
\text { - } 90 \% \text { spectral width }=33 \mathrm{GHz}\end{array}$ \\
\hline $\mathrm{RZ}$ & $\begin{array}{l}\text { - No OSNR penalty. } \\
\text { - Versatility to non-linear optical } \\
\text { fiber propagation is achieved. }\end{array}$ & $\begin{array}{l}\text { - } 66 \mathrm{GHz} \text { channel spacing is achieved } \\
\text { with ninety percentage spectral width } \\
\text {. (unfiltered), channel spacing limited } \\
\text { to } 100 \mathrm{GHz} \text {. } \\
\text { - Auxilary modulator stages are } \\
\text { required }\end{array}$ \\
\hline $\begin{array}{l}\mathrm{SCM}+ \\
\mathrm{M}-\mathrm{QAM}\end{array}$ & $\begin{array}{ll}\text { - } & \text { Sectral narrowing }=\mathrm{f}(\mathrm{M}) \\
\text { - } & \text { Symbol duration }=\mathrm{f}(\mathrm{M}) \\
& \text { Lower carrier frequency and/or } \\
\text { longer symbol duration } \\
\text { improves tolerance to } \\
\text { uncompensated CD and PMD }\end{array}$ & $\begin{array}{ll}\text { - } & \text { OSNR penalty }=\mathrm{f}(\# \text { carriers,M }) \\
\text { spectral efficiency gains more than } \\
\text { offset by large OSNR penalty } \\
\text { - } \\
\text { Requires complex analog RF } \\
\text { electronics } \\
\text { - } \\
\text { Stringent linearity requirements in } \\
\text { modulator and driver. }\end{array}$ \\
\hline DPSK & $\begin{array}{l}\text { - } 3 \text { dB OSNR improvement (with } \\
\text { balanced receiver) } \\
\text { - Constant envelope modulation }\end{array}$ & $\begin{array}{l}\text { - Interferometric detection required. } \\
\text { - Requires DPSK receiver optical } \\
\text { channel monitoring in optical line } \\
\text { system. }\end{array}$ \\
\hline
\end{tabular}


International Journal of Computer Networks \& Communications (IJCNC) Vol.6, No.2, March 2014

\begin{tabular}{|c|c|c|}
\hline & decreases SPM,XPM & \\
\hline DQPSK & $\begin{array}{l}\text { - } \\
\text { - } \quad \text { Decrease in cross polarization } \\
\text { modulation by employing } \\
\text { constant envelope modulation. } \\
\text { - } 33 \mathrm{GHz} \text { channel spacing is } \\
\text { achieved with ninety percentage } \\
\text { spectral width. }\end{array}$ & $\begin{array}{l}\text { - } \text { Interferometric detection required } \\
\text { - } \quad \text { Requires complicated drive signal or } \\
\text { - } 2 \text { modulators } \\
\text { optical channel monitoring in optical } \\
\text { line system. }\end{array}$ \\
\hline
\end{tabular}

Table 4. . Comparison of FOC Digital Modulation Spectral Efficiency and Modulation Efficiency

\begin{tabular}{|l|l|l|l|l|l|l|}
\hline $\begin{array}{l}\text { Digital } \\
\text { Modulation } \\
\text { Techniques } \\
\leq \mathbf{1 0 0 G} / \mathbf{s}\end{array}$ & $\begin{array}{l}\text { Data } \\
\text { Rate }\end{array}$ & $\begin{array}{l}\text { Number } \\
\text { of } \\
\text { Channels }\end{array}$ & $\begin{array}{l}\text { Channel } \\
\text { Spacing }\end{array}$ & $\begin{array}{l}\text { Spectral } \\
\text { Efficiency } \\
\text { (bits/s)/Hz }\end{array}$ & $\begin{array}{l}\text { Modulation } \\
\text { Efficiency } \\
\text { (Bits/Baud) }\end{array}$ & $\begin{array}{l}\text { Effective } \\
\text { Baud Rate } \\
\text { (Symbol } \\
\text { Rate) }\end{array}$ \\
\hline NRZ-00K & 10 & 40 & 100 & 0.1 & 1 & $100 \mathrm{G}$ \\
\hline DPSK & 40 & 40 & 100 & 0.4 & 1 & $100 \mathrm{G}$ \\
\hline QPSK & 10 & 80 & 50 & 0.2 & 2 & $50 \mathrm{G}$ \\
\hline DPSK-3ASK & 100 & 40 & 100 & 1 & 2.5 & 40G \\
\hline PM-QPSK & 100 & 80 & 50 & 2 & 4 & $25 \mathrm{G}$ \\
\hline
\end{tabular}

Table 5. Parametric comparison of fiber optics digital modulation formats for $400 \mathrm{~Gb} / \mathrm{s}$.

\begin{tabular}{|l|l|l|l|l|l|}
\hline $\begin{array}{l}\text { Digital } \\
\text { Modulation }\end{array}$ & Points & Symbols & $\begin{array}{l}\text { Information } \\
\text { capacity }\end{array}$ & $\begin{array}{l}\text { Derived } \\
\text { form }\end{array}$ & BW efficiency \\
\hline BASK & 01 & 01 & Poor & ASK & Poor \\
\hline BFSK & 01 & 01 & $\begin{array}{l}\text { Better than } \\
\text { BASK }\end{array}$ & FSK & Not efficient \\
\hline BPSK & 02 & 02 & 2 BFSK & PSK & Only for high speed data \\
\hline QPSK & 04 & 04 & 2BFSK & PSK & High \\
\hline MSK & 04 & 04 & 2BFSK & OQPSK & Lower than QPSK \\
\hline QAM & 02 & 04 & $\begin{array}{l}\text { Better than } \\
\text { BASK }\end{array}$ & $\begin{array}{l}\text { ASK \& } \\
\text { PSK }\end{array}$ & $\begin{array}{l}\text { Less than other } \\
\text { techniques }\end{array}$ \\
\hline 16 QAM & 04 & 04 & $\begin{array}{l}\text { Better than } \\
\text { OAM }\end{array}$ & $\begin{array}{l}\text { ASK \& } \\
\text { PSK }\end{array}$ & $\begin{array}{l}\text { Less than other } \\
\text { techniques }\end{array}$ \\
\hline 64 QAM & 06 & 04 & $\begin{array}{l}\text { Better than } \\
\text { QAM }\end{array}$ & $\begin{array}{l}\text { ASK \& } \\
\text { PSK }\end{array}$ & $\begin{array}{l}\text { Less than other } \\
\text { techniques }\end{array}$ \\
\hline GMSK & 04 & 04 & Same as & FSK & Excellent \\
\hline
\end{tabular}


International Journal of Computer Networks \& Communications (IJCNC) Vol.6, No.2, March 2014

Table 6. The standardized voice-band data modems with duplex methods for different DFOC

\begin{tabular}{|l|l|l|l|l|}
\hline $\begin{array}{l}\text { Digital } \\
\text { Modulation } \\
\text { Techniques }\end{array}$ & Speed (b/s) & $\begin{array}{l}\text { Symbol rate } \\
(\mathbf{H z})\end{array}$ & Duplex method & CCITT standard \\
\hline 2-FSK & $\leq 300$ & $\leq 300$ & Full FDM & V.21 \\
\hline 2-FSK & 1200 & 1200 & Half & V.23 \\
\hline 4-PSK & 1200 & 600 & Full FDM & V.22 \\
\hline 4-PSK & 2400 & 1200 & Half & V.26 \\
\hline 16-QAM & 2400 & 600 & Full FDM & V.22bis \\
\hline 4-PSK & 2400 & 1200 & Full-EC & V.26ter \\
\hline 8-PSK & 4800 & 1600 & Half & V.27 \\
\hline 4-QPSK & 4800 & 2400 & Full-EC & V.32 \\
\hline 16-AM/PM & 9600 & 2400 & Half & V.29 \\
\hline $\begin{array}{l}32-\text { QAM }+ \\
\text { TC }\end{array}$ & 9600 & 2400 & Full-EC & V.32 \\
\hline $\begin{array}{l}\text { 1024- QAM + } \\
\text { TC }\end{array}$ & $\leq 28,800$ & $\leq 3429$ & Full-EC & V.fast (V.34) \\
\hline
\end{tabular}

Table 7. Major parameters of modulation methods at $400 \mathrm{~Gb} / \mathrm{s}$.

\begin{tabular}{|l|l|l|l|l|l|l|l|l|}
\hline $\begin{array}{l}\text { Digital } \\
\text { Modulation } \\
\text { Formats }\end{array}$ & OOK & $\begin{array}{l}\text { OOK- } \\
\text { VSB }\end{array}$ & DQPSK & $\begin{array}{l}\text { RZ- } \\
\text { DPSK- } \\
\text { 3ASK }\end{array}$ & $\begin{array}{l}\text { PM- } \\
\text { DQPSK }\end{array}$ & $\begin{array}{l}\text { OP- } \\
\text { FDM- } \\
\text { RZ- } \\
\text { DQPSK }\end{array}$ & $\begin{array}{l}\text { PM- } \\
\text { QPSK }\end{array}$ & $\begin{array}{l}\text { PM- } \\
\text { OFD } \\
\text { M- } \\
\text { QPS } \\
\text { K }\end{array}$ \\
\hline Symbol rate & 112 & 112 & 56 & 44 & 28 & 28 & 28 & 14 \\
\hline Bits/ Symbol & 01 & 01 & 02 & 2.5 & $2 \times 2$ & $2 \times 2$ & $2 \times 2$ & $\begin{array}{l}2 \times 2 x \\
2\end{array}$ \\
\hline $\begin{array}{l}\text { Estimated } \\
\text { Reach (km) }\end{array}$ & $<500$ & $<500$ & 1000 & $<500$ & 600 & 1500 & 1500 & 2000 \\
\hline $\begin{array}{l}\text { Spectral } \\
\text { Efficiency }\end{array}$ & 0.5 & 01 & 01 & 02 & 02 & 01 & 02 & 02 \\
\hline $\begin{array}{l}\text { CD tolerance } \\
\text { ps/nm) @ 2dB } \\
\text { penalty }\end{array}$ & \pm 5 & \pm 5 & \pm 20 & \pm 30 & \pm 90 & \pm 90 & $>>$ & $>>$ \\
\hline $\begin{array}{l}\text { OSNR } \\
\text { tolerance(dB) @ } \\
\text { BER 4x 10 }\end{array}$ & 17.5 & 18.5 & 15.5 & $>20$ & 15.5 & 15.5 & $<15$ & $<15$ \\
\hline $\begin{array}{l}\text { Coherent/ Non- } \\
\text { coherent }\end{array}$ & $\begin{array}{l}\text { Non- } \\
\text { cohent }\end{array}$ & $\begin{array}{l}\text { Non- } \\
\text { coherent }\end{array}$ & $\begin{array}{l}\text { Non- } \\
\text { cohent }\end{array}$ & $\begin{array}{l}\text { Non- } \\
\text { coherent }\end{array}$ & $\begin{array}{l}\text { Non- } \\
\text { coherent }\end{array}$ & $\begin{array}{l}\text { Non- } \\
\text { coherent }\end{array}$ & Coherent & $\begin{array}{l}\text { Cohe } \\
\text { rent }\end{array}$ \\
\hline $\begin{array}{l}\text { Product } \\
\text { Available }\end{array}$ & No & No & No & No & No & Yes & Yes & Yes \\
\hline
\end{tabular}


International Journal of Computer Networks \& Communications (IJCNC) Vol.6, No.2, March 2014

Table 8. Analysis of various digital modulation methods up to $1000 \mathrm{~Gb} / \mathrm{s}$ with theoretical value of $40 \mathrm{~Gb} / \mathrm{s}$ taken as reference.

\begin{tabular}{|l|l|l|l|l|l|l|l|l|}
\hline $\begin{array}{l}\text { Digital } \\
\text { Modulation } \\
\text { Formats }\end{array}$ & $\begin{array}{l}\text { PM- } \\
\text { BPS } \\
\text { K }\end{array}$ & $\begin{array}{l}\text { PM- } \\
\text { QPSK }\end{array}$ & $\begin{array}{l}\text { PM-8 } \\
\text { QAM }\end{array}$ & $\begin{array}{l}\text { PM- } \\
\text { 16 } \\
\text { QAM }\end{array}$ & $\begin{array}{l}\text { PM- } \\
\text { 32 } \\
\text { QAM }\end{array}$ & $\begin{array}{l}\text { PM- } \\
\text { 32 } \\
\text { QAM }\end{array}$ & $\begin{array}{l}\text { PM- } \\
\text { 64 } \\
\text { QAM }\end{array}$ & $\begin{array}{l}\text { PM- } \\
\text { 256- } \\
\text { QAM }\end{array}$ \\
\hline $\begin{array}{l}\text { Channel } \\
\text { Spacing }\end{array}$ & 50 & 200 & 133 & 100 & 80 & 200 & 67 & 50 \\
\hline Bit-Rate (Gb/s) & 100 & 400 & 400 & 400 & 400 & 1000 & 400 & 400 \\
\hline Bits/Symbol & $2 \times 1$ & $2 \times 2$ & $2 \times 3$ & $2 \times 4$ & $2 \times 5$ & $2 \times 5$ & $2 \times 6$ & $2 \times 8$ \\
\hline $\begin{array}{l}\text { Symbol Rate } \\
28-32\end{array}$ & $\begin{array}{l}112- \\
128\end{array}$ & $75-85$ & $56-64$ & $45-51$ & $\begin{array}{l}112- \\
128\end{array}$ & $37-43$ & $28-32$ \\
\hline $\begin{array}{l}\text { Penalty vs 100G } \\
\text { (dB) }\end{array}$ & 00 & 06 & 08 & 10 & 12 & 16 & 14.5 & $>20$ \\
\hline $\begin{array}{l}\text { No. of C-Band } \\
\text { Channels }\end{array}$ & 44 & 22 & 33 & 44 & 55 & 22 & 66 & 88 \\
\hline $\begin{array}{l}\text { Total Capacity } \\
\text { (Tb/s) }\end{array}$ & 8.8 & 8.8 & 13.3 & 17.6 & 22 & 22 & 26.4 & 35 \\
\hline $\begin{array}{l}\text { OSNR (dB) @ } \\
\text { Min. Baud Rate }\end{array}$ & 10.8 & 18.2 & 20.2 & 22.2 & 24.2 & 28.2 & 26.7 & $>30$ \\
\hline $\begin{array}{l}\text { OSNR (dB) @ } \\
\text { Max. Baud Rate }\end{array}$ & 8.2 & 15.8 & 17.8 & 19.8 & 21.8 & 25.8 & 24.3 & $>32$ \\
\hline
\end{tabular}

Table 9. Transmission rate performance comparison for NRZ fiber modulation coding format within $400 \mathrm{~Gb} / \mathrm{s}$.

\begin{tabular}{|c|c|c|c|c|c|c|c|}
\hline $\begin{array}{l}\text { Channel } \\
\text { Bit Rate }\end{array}$ & \begin{tabular}{|l} 
Multiplexin \\
g \\
Method
\end{tabular} & $\begin{array}{l}\text { PMD } \\
\text { delay } \\
\text { (pico- }\end{array}$ & \begin{tabular}{|l} 
Maximum \\
Dispersio \\
at 1550
\end{tabular} & $\begin{array}{l}\text { Insertion } \\
\text { Loss }\end{array}$ & $\begin{array}{l}\text { Retur } \\
\text { n } \\
\text { Loss }\end{array}$ & \begin{tabular}{|l} 
Physical \\
plant \\
verification
\end{tabular} & $\begin{array}{l}\text { Attenuation } \\
\text { Profile }\end{array}$ \\
\hline $\begin{array}{l}2.5 \mathrm{Gbps} \\
\text { DWDM }\end{array}$ & \begin{tabular}{|l|} 
OC- \\
48/STM-16
\end{tabular} & 40 & 18817 & $\begin{array}{l}1550 / 162 \\
5\end{array}$ & $\begin{array}{l}1550 \\
\mathrm{~nm}\end{array}$ & $\begin{array}{l}1550 / 1625 \\
\mathrm{~nm}\end{array}$ & $\begin{array}{l}1550- \\
1625 \mathrm{~nm}\end{array}$ \\
\hline $\begin{array}{l}10 \text { Gbps } \\
\text { DWDM }\end{array}$ & $\begin{array}{l}\text { OC- } \\
\text { 192/STM-64 }\end{array}$ & 10 & 1176 & $\frac{1550 / 162}{5}$ & $\begin{array}{l}1550 \\
\mathrm{~nm}\end{array}$ & $\begin{array}{l}1550 / 1625 \\
\mathrm{~nm}\end{array}$ & $\begin{array}{l}1550- \\
1625 \mathrm{~nm}\end{array}$ \\
\hline $\begin{array}{l}40 \text { Gbps } \\
\text { DWDM }\end{array}$ & $\begin{array}{l}\text { OC- } \\
\text { 768/STM- }\end{array}$ & 2.5 & 73.5 & $\begin{array}{l}\text { nm } \\
1310 / 155 \\
0\end{array}$ & $\begin{array}{l}1550 \\
\mathrm{~nm}\end{array}$ & $\begin{array}{l}1310 / 1550 \\
\mathrm{~nm}\end{array}$ & \begin{tabular}{|l|}
$1550-$ \\
$1625 \mathrm{~nm}$
\end{tabular} \\
\hline $10 \mathrm{Gbps}$ & Ethernet & 5 & 738 & $\begin{array}{l}1310 / 155 \\
0\end{array}$ & $\begin{array}{l}1550 \\
\mathrm{~nm}\end{array}$ & $\begin{array}{l}1310 / 1550 \\
\mathrm{~nm}\end{array}$ & $\begin{array}{l}1550- \\
1625 \mathrm{~nm}\end{array}$ \\
\hline
\end{tabular}

Table 10. Performances and complexity Comparison between different multiplexing techniques and modulation formats at $400 \mathrm{~Gb} / \mathrm{s}$ aggregation bit-rate.

\begin{tabular}{|c|c|c|c|c|c|c|}
\hline $\begin{array}{l}\text { Digital } \\
\text { Modulation } \\
\& \\
\text { Multiplexing } \\
\text { techniques }\end{array}$ & $\begin{array}{l}\text { Transmitte } \\
\mathbf{r} \\
\mathbf{T}_{\mathbf{X}}\end{array}$ & $\begin{array}{l}\text { Receive } \\
\mathbf{r} \\
\mathbf{R}_{\mathbf{X}}\end{array}$ & OSNR (dBm) & $\begin{array}{l}\text { CD } \\
\text { (PS/n } \\
\text { m) }\end{array}$ & $\begin{array}{l}\text { MBW } \\
\text { (Ps/nm } \\
\text { ) }\end{array}$ & $\begin{array}{l}\text { CRF } \\
\text { (GHz } \\
\text { ) }\end{array}$ \\
\hline NRZ-WDM & $1 \mathrm{M}$ & $1 \mathrm{PD}$ & $\begin{array}{l}\text { Sim : } 16.5(\mathrm{E}-3) \\
19.8(\mathrm{E}-9) \\
\text { Exp: } \approx 23.3(\mathrm{E}-9)\end{array}$ & 54 & 80 & 40 \\
\hline
\end{tabular}


International Journal of Computer Networks \& Communications (IJCNC) Vol.6, No.2, March 2014

\begin{tabular}{|c|c|c|c|c|c|c|}
\hline $\begin{array}{l}50 \% \text { RZ- } \\
\text { WDM }\end{array}$ & $2 \mathrm{Ms}$ & ? & $\begin{array}{l}\text { Sim : } 14.4(\mathrm{E}-3) \\
18.3(\mathrm{E}-9) \\
\text { Exp: } \approx 21(\mathrm{E}-9)\end{array}$ & 48 & 160 & 40 \\
\hline DB & $?$ & $?$ & Sim : 22.4 (E-9) & $?$ & 40 & ? \\
\hline NRZ-DPSK & $1 \mathrm{M}$ & $\begin{array}{l}1 \mathrm{DI}+ \\
2 \mathrm{PDs}\end{array}$ & $\begin{array}{l}\text { Sim : } 11.7(\text { E-3), } \\
13.5(\text { E-3) } \\
\text { Exp: } \approx 20(\text { E-9) }\end{array}$ & 74 & 80 & 40 \\
\hline $\begin{array}{l}\text { NRZ-16- } \\
\text { QAM }\end{array}$ & 3PCs, $1 \mathrm{M}$ & $\begin{array}{l}\text { 2PDs,3P } \\
\text { Cs,POI, } \\
\text { TFL }\end{array}$ & Sim : 20.9 (E-9) & $?$ & 20 & 10 \\
\hline $\begin{array}{l}\text { E-DCDM } \\
(2 X 20 \mathrm{~Gb} / \mathrm{s})\end{array}$ & $1 \mathrm{M}$ & 1PD & $\begin{array}{l}\text { Sim : } 17.8(\mathrm{E}-3) \\
21.74(\mathrm{E}-9)\end{array}$ & 62 & 120 & 20 \\
\hline $\begin{array}{l}\text { E-DCDM } \\
(4 \mathrm{X} 10 \mathrm{~Gb} / \mathrm{s})\end{array}$ & $1 \mathrm{M}$ & 1PD & $\begin{array}{l}\text { Sim : } 21.6 \text { (E-3) } \\
26.4(E-9)\end{array}$ & 58 & 100 & 10 \\
\hline $\begin{array}{l}\text { E-DCDM } \\
(7 X 5.71 \mathrm{~Gb} / \mathrm{s})\end{array}$ & $1 \mathrm{M}$ & 1PD & $\begin{array}{l}\text { Sim : } 27 \text { (E-3) } \\
31.4(E-9)\end{array}$ & 52 & 91.4 & 5.71 \\
\hline
\end{tabular}

Table 11. The proposed ITU-standard for DWDM channel codes.

\begin{tabular}{|c|c|c|c|c|c|c|c|}
\hline $\begin{array}{l}\text { DWDM } \\
\text { Channel } \\
\text { Code }\end{array}$ & $\begin{array}{l}\lambda \\
(\mathrm{nm})\end{array}$ & $\begin{array}{l}\text { DWDM } \\
\text { Channel } \\
\text { Code }\end{array}$ & $\begin{array}{l}\lambda \\
(\mathbf{n m})\end{array}$ & $\begin{array}{l}\text { DWDM } \\
\text { Channel } \\
\text { Code }\end{array}$ & $\begin{array}{l}\lambda \\
(\mathrm{nm})\end{array}$ & $\begin{array}{l}\text { DWDM } \\
\text { Channel } \\
\text { Code }\end{array}$ & $\begin{array}{l}\lambda \\
(\mathbf{n m})\end{array}$ \\
\hline 18 & 1563.05 & 30 & 1553.33 & 42 & 1543.73 & 54 & 1534.25 \\
\hline 19 & 1562.23 & 31 & 1552.53 & 43 & 1542.94 & 55 & 1533.47 \\
\hline 20 & 1561.42 & 32 & 1551.72 & 44 & 1542.14 & 56 & 1532.68 \\
\hline 21 & 1560.61 & 33 & 1550.92 & 45 & 1541.35 & 57 & 1531.90 \\
\hline 22 & 1559.80 & 34 & 1550.12 & 46 & 1540.56 & 58 & 1531.12 \\
\hline 23 & 1558.98 & 35 & 1549.32 & 47 & 1539.77 & 59 & 1530.33 \\
\hline 24 & 1558.17 & 36 & 1548.52 & 48 & 1538.98 & 60 & 1529.55 \\
\hline 25 & 1557.36 & 37 & 1547.72 & 49 & 1538.19 & 61 & 1528.77 \\
\hline 26 & 1556.56 & 38 & 1546.92 & 50 & 1537.40 & 62 & 1527.99 \\
\hline 27 & 1555.75 & 39 & 1546.12 & 51 & 1536.61 & & \\
\hline 28 & 1554.94 & 40 & 1545.32 & 53 & 1535.82 & & \\
\hline 29 & 1554.13 & 41 & 1544.53 & 53 & 1535.04 & & \\
\hline
\end{tabular}


International Journal of Computer Networks \& Communications (IJCNC) Vol.6, No.2, March 2014

Table 12. Optical OFDM Parameters for $100 \mathrm{~Gb} / \mathrm{s}$ using Polarization-multiplexed QAM.

\begin{tabular}{|c|c|c|c|c|c|c|c|c|c|}
\hline \multirow{3}{*}{$\begin{array}{l}\text { Transmission } \\
\text { Distance } \\
\text { (Km) }\end{array}$} & \multicolumn{9}{|c|}{ Polarization-Multiplexed Transmission } \\
\hline & \multicolumn{3}{|c|}{$\begin{array}{l}\text { 4-QAM } \\
\text { (4 bits/symbol) }\end{array}$} & \multicolumn{3}{|c|}{$\begin{array}{l}\text { 8-QAM } \\
(6 \text { bits/symbol })\end{array}$} & \multicolumn{3}{|c|}{$\begin{array}{l}\text { 16-QAM } \\
(8 \mathrm{bits} / \mathrm{symbol})\end{array}$} \\
\hline & Npre & Nc & $\mathbf{N u}$ & Npre & $\mathrm{Ne}$ & $\mathrm{Nu}$ & Npre & Nc & $\mathrm{Nu}$ \\
\hline 1000 & 5 & 32 & 26 & 4 & 32 & 26 & 2 & 16 & 13 \\
\hline 2000 & 8 & 64 & 52 & 5 & 32 & 26 & 4 & 32 & 26 \\
\hline 3000 & 10 & 64 & 52 & 6 & 32 & 26 & 5 & 32 & 26 \\
\hline 5000 & 14 & 128 & 104 & 8 & 64 & 52 & 6 & 32 & 26 \\
\hline
\end{tabular}

Table 13. Polarization multiplexed complexity of single-carrier transmission compared to Optical Orthogonal Frequency Division Multiplexing at 400Gb/s.

\begin{tabular}{|l|l|l|l|l|l|l|}
\hline \multirow{2}{*}{$\begin{array}{l}\text { Transmission } \\
\text { Distance (Km) }\end{array}$} & \multicolumn{2}{|l|}{ Single-Carrier } & \multicolumn{2}{l|}{ OFDM } \\
\cline { 2 - 7 } & \multirow{2}{*}{\begin{tabular}{l} 
Direct \\
\cline { 3 - 7 }
\end{tabular}} & \begin{tabular}{l} 
FFT \\
Block \\
\cline { 2 - 7 }
\end{tabular} & Complexity & Transmitter & Receiver & Total \\
\hline 1000 & 12.0 & 6 & 13.3 & 6.2 & 10.2 & 16.4 \\
\hline 2000 & 24.0 & 27 & 16.6 & 7.4 & 11.4 & 18.8 \\
\hline 3000 & 32.0 & 25 & 17.9 & 7.4 & 11.4 & 18.8 \\
\hline 5000 & 52.0 & 52 & 19.7 & 8.6 & 12.6 & 21.2 \\
\hline
\end{tabular}

Table 14. Performance Comparison of CWDM and DWDM technology at 400Gb/s of WDM.

\begin{tabular}{|l|l|l|}
\hline $\begin{array}{l}\text { Features of WDM in } \\
\text { DFOC }\end{array}$ & Coarse WDM & Dense WDM \\
\hline Laser Transmitter types & Uncooled DFB & $\begin{array}{l}\text { Cooled DFB, external } \\
\text { modulation }\end{array}$ \\
\hline Spacing of wavelentghs & $2500 \mathrm{GHz}(20 \mathrm{~nm})$ & $100 \mathrm{GHz}(0.8 \mathrm{~nm})$ \\
\hline Wavelenghts/ fiber $(\lambda)$ & $8-16(\mathrm{O}, \mathrm{E}, \mathrm{S}, \mathrm{C}, \mathrm{L}$ bands $)$ & $40-80(\mathrm{C}, \mathrm{L}$ bands $)$ \\
\hline $\begin{array}{l}\text { Capacity of each } \\
\text { wavelength }\end{array}$ & Up to $2.5 \mathrm{Gbps}$ & Up to $10 \mathrm{Gbps}$ \\
\hline Total Capacity & $20-40 \mathrm{Gbps}$ & $100-1000 \mathrm{Gbps}$ \\
\hline $\begin{array}{l}\text { Fiber Technology } \\
\text { Thin film }\end{array}$ & $\begin{array}{l}\text { Thin film, AWG, Bragg } \\
\text { grating }\end{array}$ \\
\hline Transmission distances & Up to 70 KM & Up to 900 KM \\
\hline Overall Cost & Very low & Medium \\
\hline
\end{tabular}


International Journal of Computer Networks \& Communications (IJCNC) Vol.6, No.2, March 2014

\begin{tabular}{|l|l|l|}
\hline Application & Enterprise, metro-access & Access, metro-core, regional \\
\hline Transmitter Board Area & $20 \mathrm{~cm}^{2}\left(3.1 \mathrm{in}^{2}\right)$ & $100 \mathrm{~cm}^{2}\left(16 \mathrm{in}^{2}\right)$ \\
\hline $\begin{array}{l}\text { Power Consumption per Tx } \\
\text { Card (SDI) }\end{array}$ & $1.6 \mathrm{~W}(100 \mathrm{GHz})$ & $5 \mathrm{~W}$ typically $(100 \mathrm{GHz})$ \\
\hline $\begin{array}{l}\text { Laser Wavelength variation } \\
\left(0-40^{\circ} \mathrm{C}\right)\end{array}$ & $\pm 6.5 \mathrm{~nm}$ & $\pm 0.16 \mathrm{~nm}$ \\
\hline Channel Spacing & $20 \mathrm{~nm}$ & $0.8 \mathrm{~nm}$ \\
\hline Channels per frame & $4+1$ upgrade port & $4+1$ upgrade port \\
\hline Wavelength Selection & Standard ITU wavelength & Reduced \\
\hline Raman Crosstalk & $\begin{array}{l}\text { Significant without } \\
\text { mitigation techniques }\end{array}$ & $\begin{array}{l}\text { Minimal with selective } \\
\text { wavelength spacing }\end{array}$ \\
\hline Four-wave mixing & Not Applicable & Not Applicable \\
\hline $\begin{array}{l}\text { Dependence on the } \\
\text { Dispersion of delayed fiber }\end{array}$ & Low dependence & High Dependence \\
\hline No. of Wavelengths & 2-5 & 2-8 \\
\hline
\end{tabular}

Table 15. Constellation diagrams of different FOC digital modulation formats.

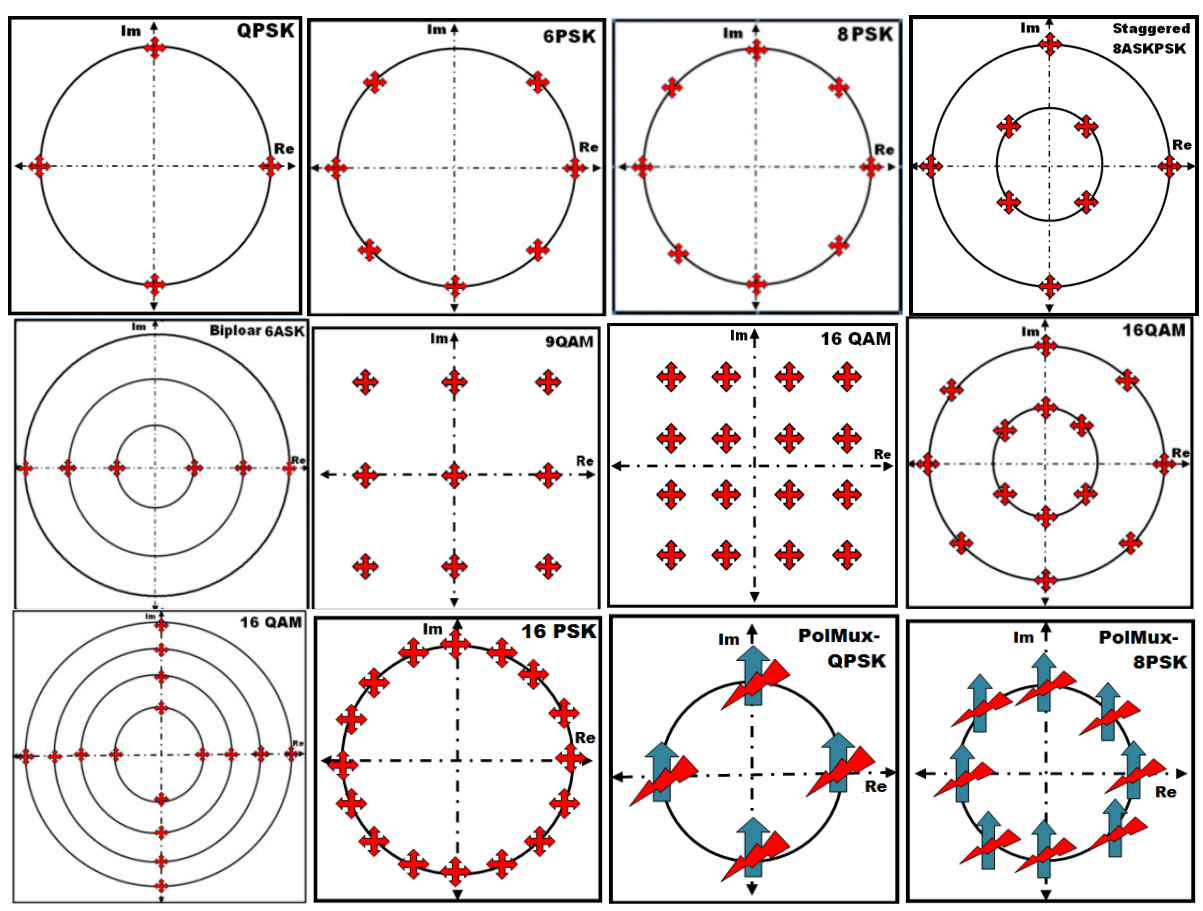


International Journal of Computer Networks \& Communications (IJCNC) Vol.6, No.2, March 2014

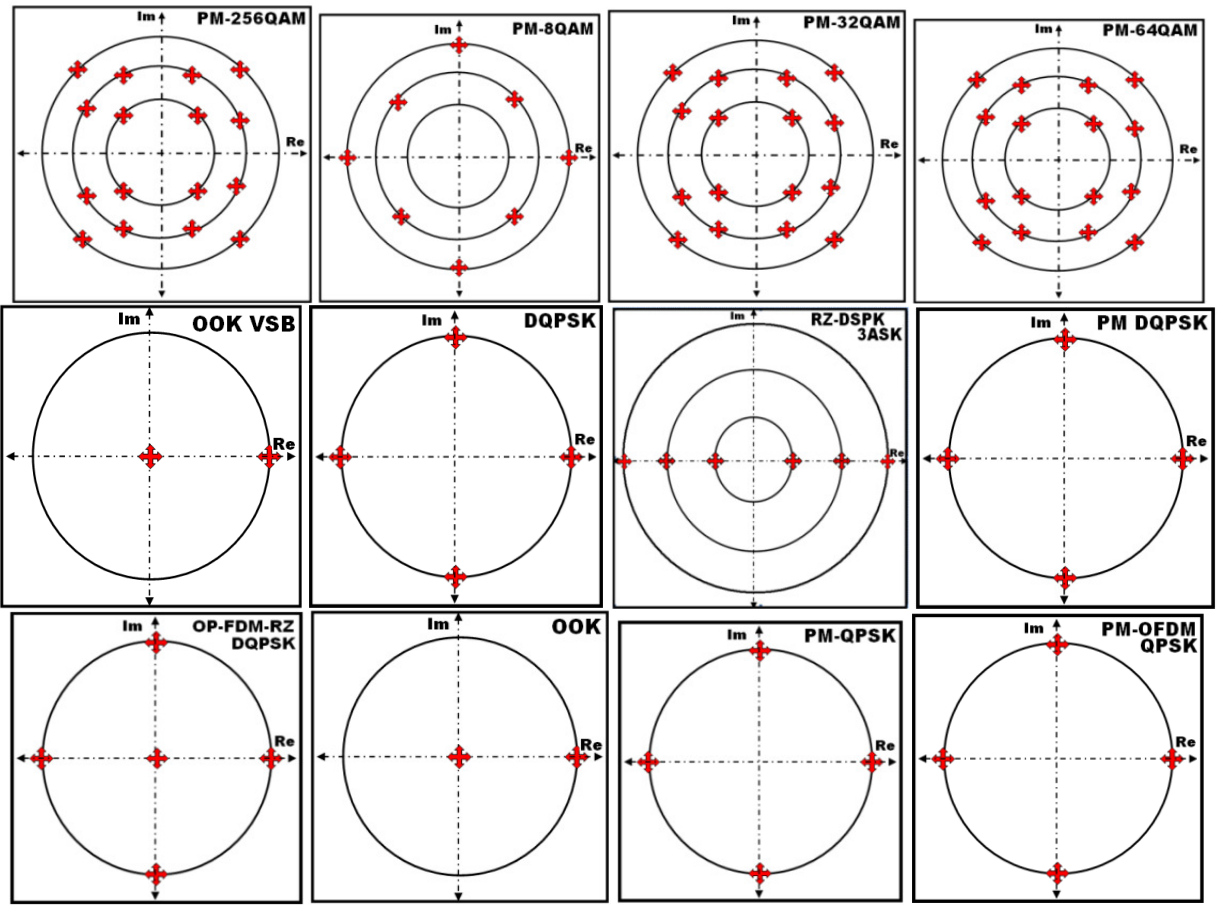

Table 16. 3D graphical comparison between different DFOC parameters within $100 \mathrm{~Gb} / \mathrm{s}$

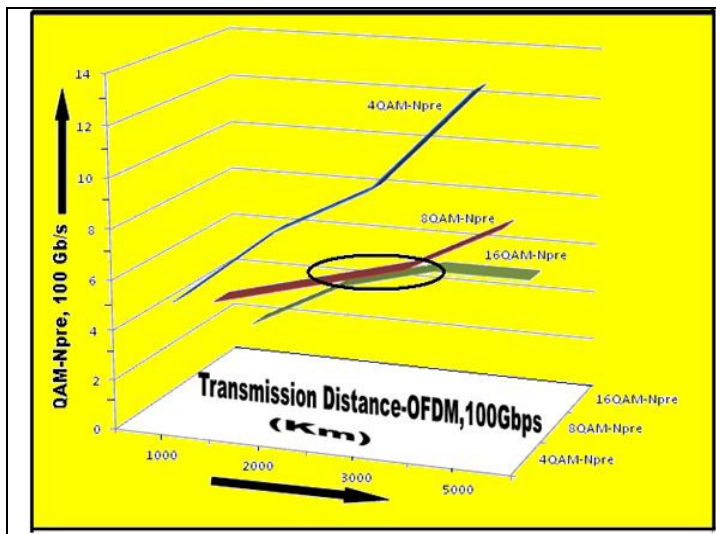

Figure 1. OFDM Performance for $100 \mathrm{~Gb} / \mathrm{s}$ Transmission using Polarization Multiplexed QAM

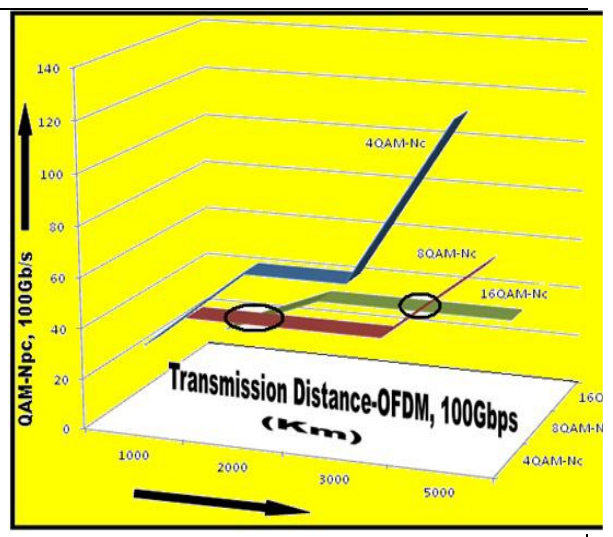

Figure 2. OFDM Performance for 100 $\mathrm{Gb} / \mathrm{s}$

Transmission using Polarization Multiplexed QAM 
International Journal of Computer Networks \& Communications (IJCNC) Vol.6, No.2, March 2014

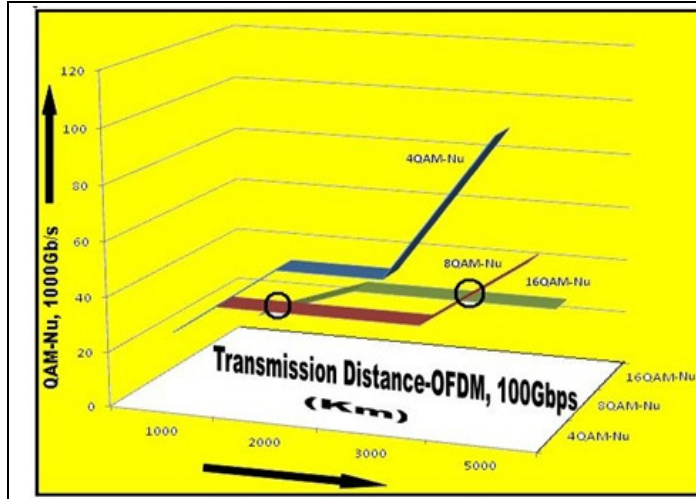

Figure 3. OFDM Performance for $100 \mathrm{~Gb} / \mathrm{s}$ Transmission using Polarization Multiplexed QAM

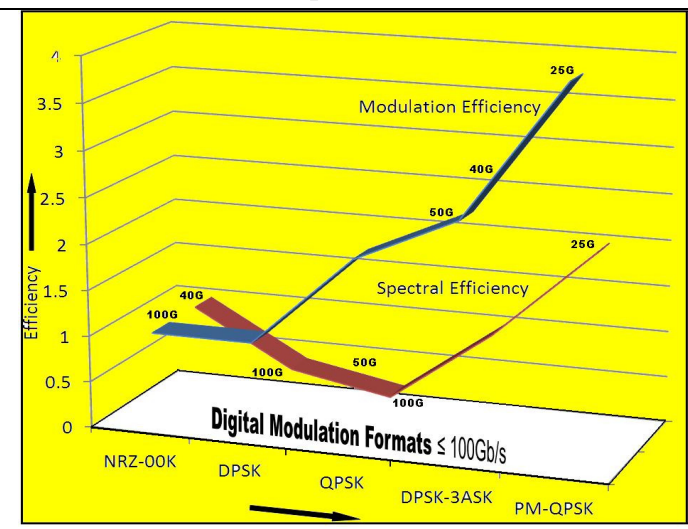

Figure 5. Spectral efficiency features w.r.t modulation efficiency for $\leq 100$ Gb/s Digital Modulation Formats.

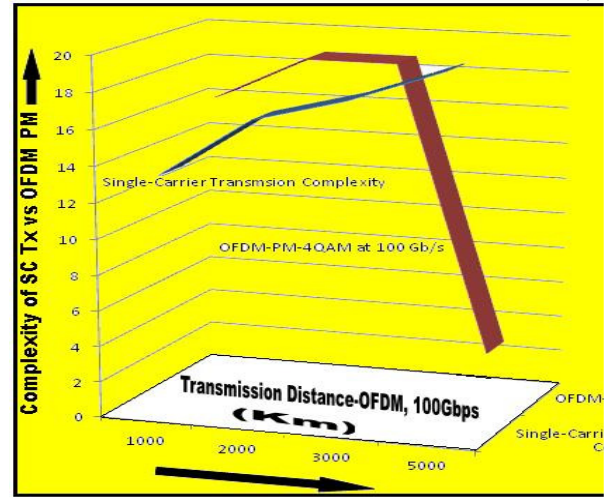

Figure 4. Computational complexity of Single-carrier transmission vs OFDM-PM-4QAM at $100 \mathrm{~Gb} / \mathrm{s}$

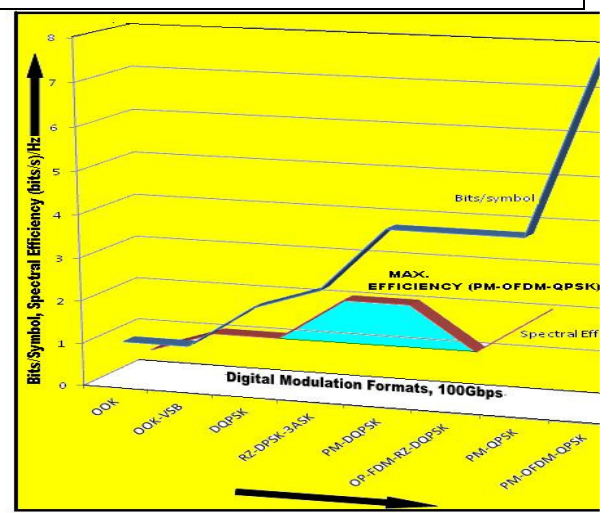

Figure 6. Spectral Efficiency features w.r.t bits/symbol for $100 \mathrm{~Gb} / \mathrm{s}$ Digital Modulation Formats.

Table 17. 3D graphical comparison between different DFOC parameters within $1 \mathrm{~Tb} / \mathrm{s}$.

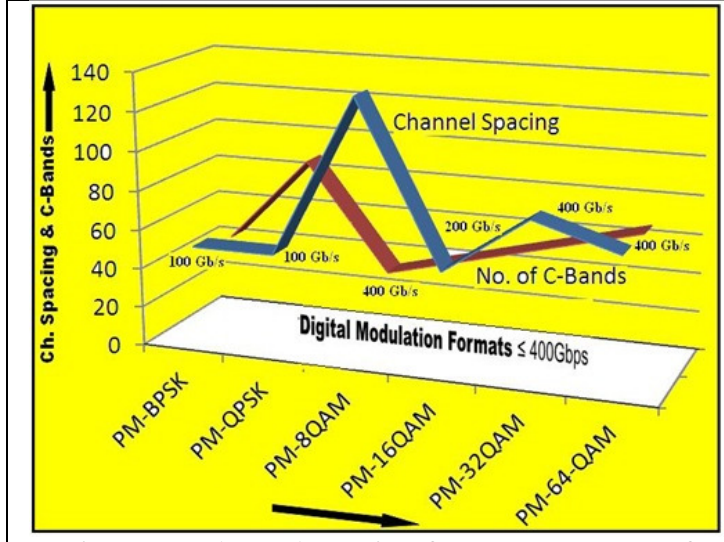

Figure 7. Channel Spacing features w.r.t no. Of C-Band $\leq 400 \mathrm{~Gb} / \mathrm{s}$ Digital Modulation Formats.

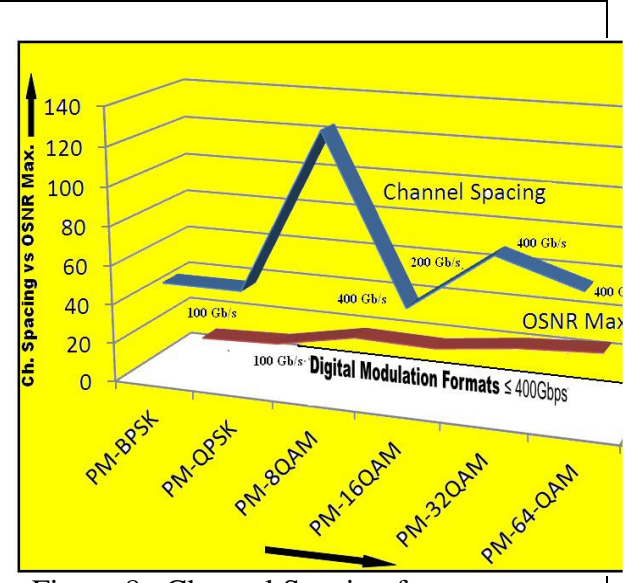

Figure 8. Channel Spacing features w.r.t OSNR (max.) for $\leq 400 \mathrm{~Gb} / \mathrm{s}$ Digital Modulation Formats. 
International Journal of Computer Networks \& Communications (IJCNC) Vol.6, No.2, March 2014

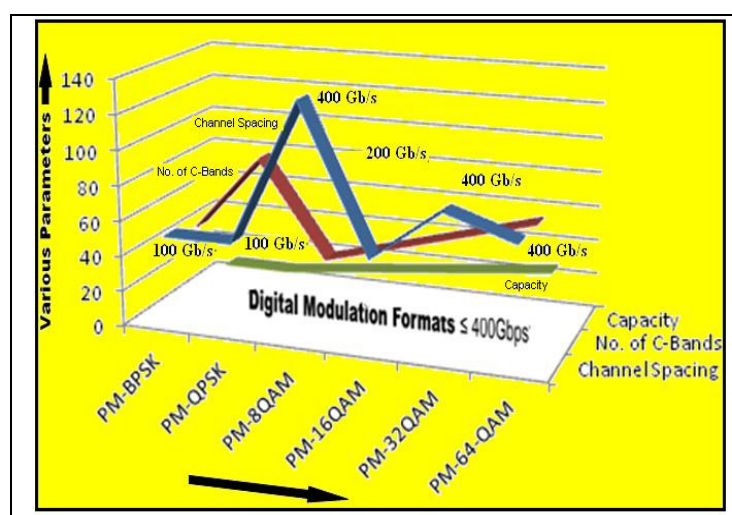

Figure 9. Channel Spacing vs No. of C-bands vs Capacity at $\leq 400 \mathrm{~Gb} / \mathrm{s}$ rate.

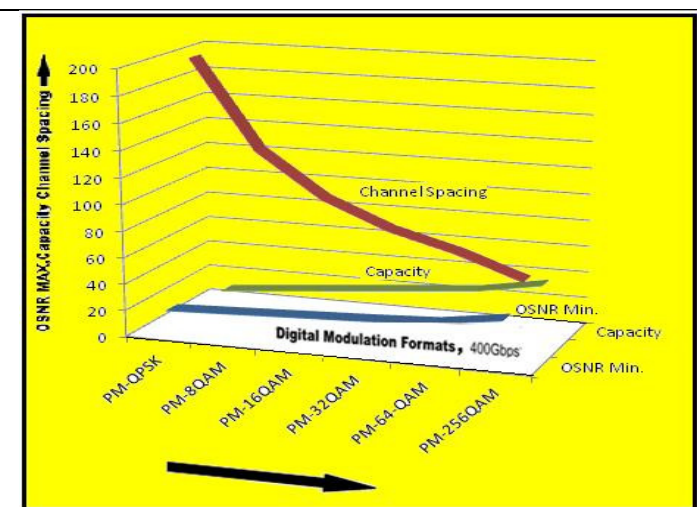

Figure 11. Channel Spacing vs OSNR (min.) vs Capacity for $\leq 400 \mathrm{~Gb} / \mathrm{s}$ Digital Modulation Formats.

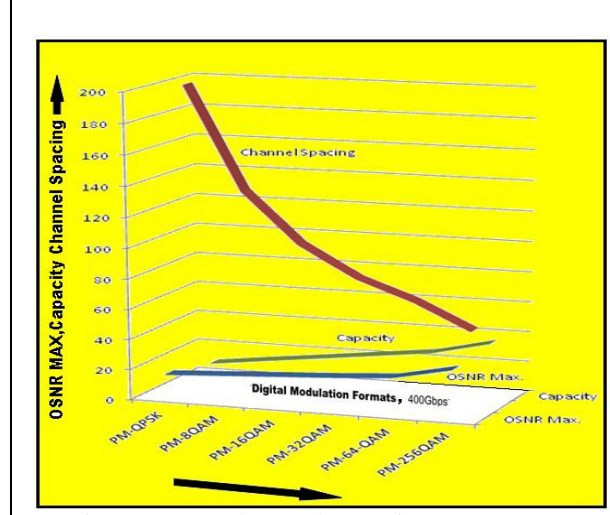

Figure 10. Channel Spacing vs OSNR (max.)

vs Capacity at $\leq 400 \mathrm{~Gb} / \mathrm{s}$

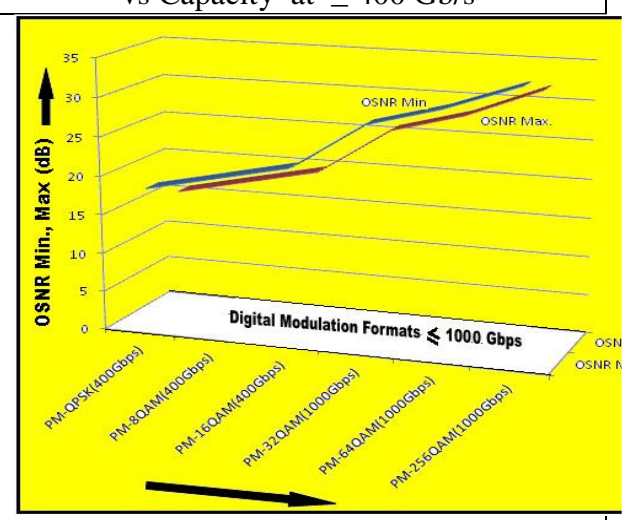

Figure 12. OSNR (max.) vs OSNR (min.) for

$\leq 1 \mathrm{~Tb} / \mathrm{s}$ Digital Modulation

Formats.

Table 18. 3D graphical comparison between different digital Modulation and Multiplexing parameters within $1 \mathrm{~Tb} / \mathrm{s}$.

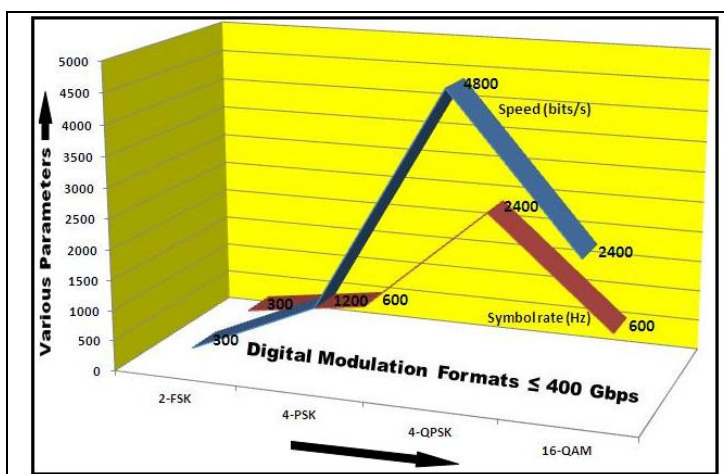

Figure 13. Speed vs symbol rate for $\leq 400$ $\mathrm{Gb} / \mathrm{s}$ Digital Modulation Formats. (Full-Duplex).

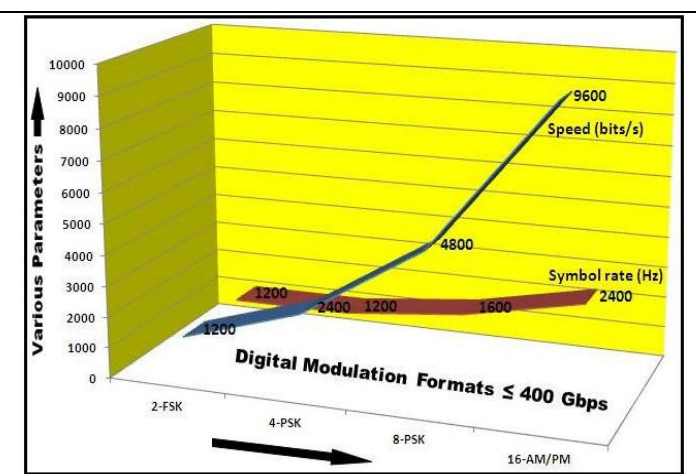

Figure 14. Speed vs symbol rate for $\leq 400$ $\mathrm{Gb} / \mathrm{s}$ Digital Modulation Formats. (Half-Duplex). 
International Journal of Computer Networks \& Communications (IJCNC) Vol.6, No.2, March 2014

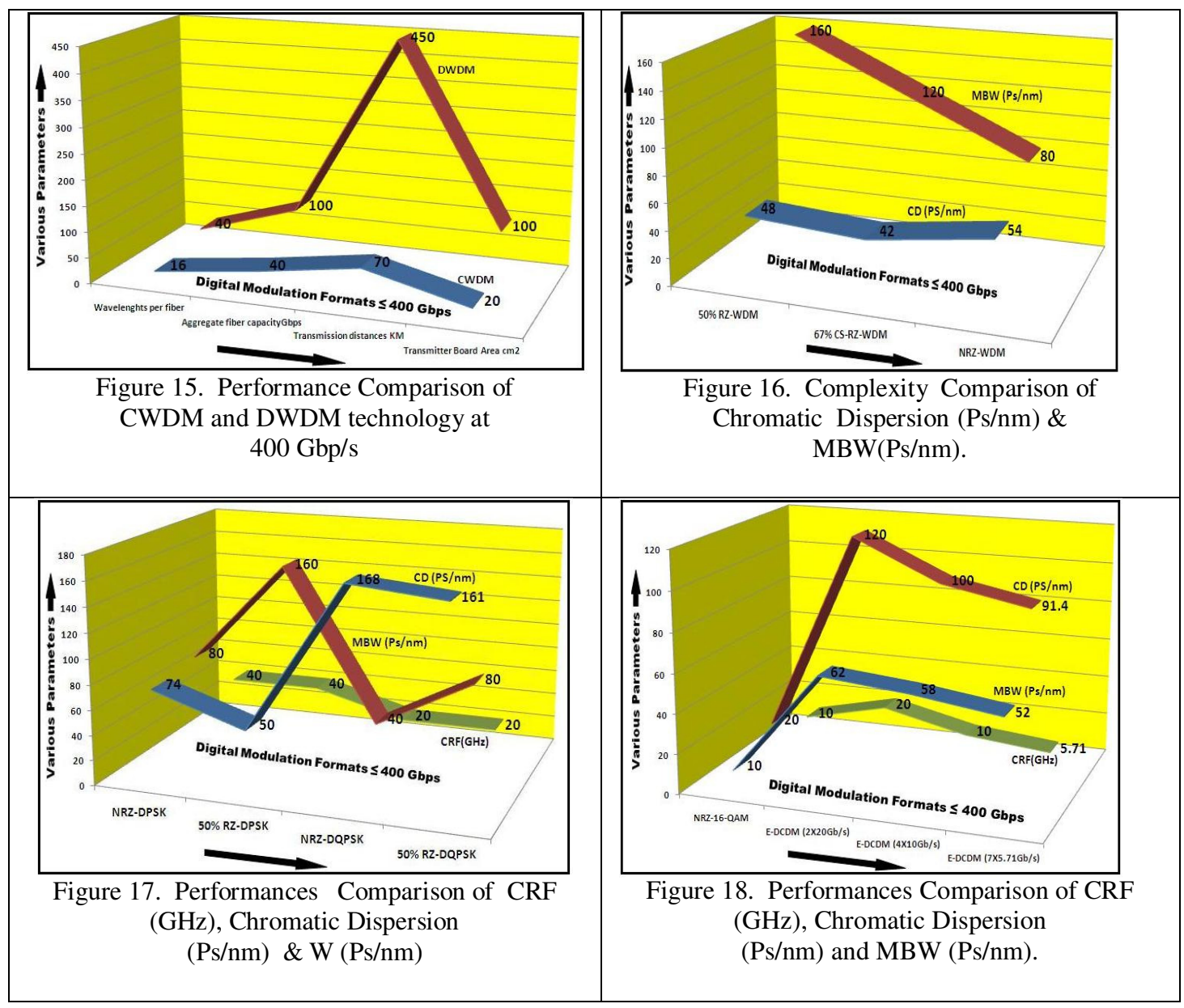

\section{Comparative Analysis}

The parameters of different types of digital fiber optic communication modulation formats with their multiplexing techniques are compared in a tabular manner from Table 1-10. Also by 3D graph representation from Table 16, $17 \& \mathbf{1 8}$, we compare the characteristics of different formats having bit-rate of $400 \mathrm{~Gb} / \mathrm{s}-1 \mathrm{~Tb} / \mathrm{s}$. The bandwidth efficiency is excellent in case of GMSK compared to BASK, BPSK, BFSK, QPSK and M-QAM modulation techniques summarized in Table-5. The modulation formats having speed (b/s) of 2-FSK $(\leq 300 \mathrm{~b} / \mathrm{s}$ and $1200 \mathrm{~b} / \mathrm{s})$, 4-PSK $(1200 \mathrm{~b} / \mathrm{s})$ up to $1024-\mathrm{QAM}+\mathrm{TC}(\leq 28,800 \mathrm{~b} / \mathrm{s})$ compared on the basis of their duplex methods used in Table-6. An comparative survey reflects in Table $7 \& 8$ on single channel M-QAM options like PM-16QAM of $200 \mathrm{~Gb} / \mathrm{s}$, PM-8QAM of 400Gb/s, PM-32-QAM of 1000Gb/s, PM$64-\mathrm{QAM}$ of $1000 \mathrm{~Gb} / \mathrm{s}, \mathrm{PM}-256-\mathrm{QAM}$ of $1000 \mathrm{~Gb} / \mathrm{s}$ by taking $40 \mathrm{~Gb} / \mathrm{s}$ value as reference, which considering Polarization multiplexing for all options. The 67\% CS-RZ-WDM and 50\% RZ-WDM utilizes two modulators as well as in 50\% RZ-DPSK shown in Table-10. The complexity between different FOC digital modulation techniques and multiplexing techniques are indicated in Table-10. The proposed $1550 \mathrm{~nm}$ window i.e DWDM by ITU is shown in Table-11. A survey of single carrier transmission vs OFDM for polarization multiplexed 4-QAM at $400 \mathrm{~Gb} / \mathrm{s}$ is summarized in Table-13. The transmission distance is up to $70 \mathrm{~km}$ (CWDM) and upto $900 \mathrm{~km}$ (DWDM) is indicated in Table-14. The 4-QAM,8-QAM \&16-QAM modulation formats having their polarization multiplexed transmission performances compares in Table-12. The Table-15 comparatively exhibits the constellation diagrams of different FOC digital modulation formats. 
International Journal of Computer Networks \& Communications (IJCNC) Vol.6, No.2, March 2014

Figure 1, 2 \& 3 of Table-16 reflects a 3D-comparative survey on OFDM performance for $100 \mathrm{~Gb} / \mathrm{s}$. A comparison between channel spacing and C-Bands of PM-BPSK, PM-QPSK, PM-MQAM having $100 \mathrm{~Gb} / \mathrm{s}$ to $400 \mathrm{~Gb} / \mathrm{s}$ is shown in figure 7 of Table-17. The OSNR at maximum range compares with channel spacing by taking different modulation techniques summarizes in a $3-\mathrm{D}$ pattern in figure $8 \& 9$ of Table-17. The symbol rate of 400-1000 Gb/s applied for comparison of OSNR-maximum \& OSNR-minimum. of different digital fiber optic communication modulation formats in figure 12 of Table-17. The comparative analysis of complexity between different WDM multiplexing techniques (50\% RZ-WDM, 67\% CS-RZWDM \& NRZ-WDM) are shown in figure 16 of Table-18. Here MBW (ps/nm) compared with chromatic dispersion at $400 \mathrm{~Gb} / \mathrm{s}$. The symbol rate varies with the bit/sec for $2 \mathrm{FSK}$, 4PSK, 4QPSK and 16-QAM at $2400 \mathrm{~Hz}$ (4800 bits/sec). Various parameters like wavelength per fiber, aggregate fiber capacity at $400 \mathrm{~Gb} / \mathrm{s}$, transmission distances and transmitter board area of CWDM and DWDM are analyzed in figure 15 of Table-18. The symbol rate vs speed for different FOC digital modulation formats are compared for half and full duplex standardized voice-band data modems.

\section{Conclusions}

In this article we describe the influence of bit rate $(\mathrm{Gb} / \mathrm{s})$ on different fiber optic communication digital modulation, detection and multiplexing techniques. We analyzed the performance of PMQPSK (100Gb/s, 400Gb/s), PM-8QAM (400Gb/s), PM-16QAM (200Gb/s), PM-16QAM (200Gb/s, 400Gb/s), PM-32QAM (400Gb/s), PM-32QAM (1000Gb/s), PM-256QAM (400Gb/s) and PM-64QAM $(1000 \mathrm{~Gb} / \mathrm{s})$. This paper not only affords simple digital modulation techniques but also provides a comparative analysis about different detection and multiplexing techniques in the optical transmission system. In this article, applications are build up using 3D practical exposure in the digital fiber optic communication.

\section{REFERENCES}

[1] K.-P. Ho, "Exact evaluation of the capacity for intensity-modulated direct-detection channels with optical mplifier noises," IEEE Photon. Technol. Lett. 17, 858-860 (2005).

[2] T. Pfau, S. Hoffmann, R. Peveling, S. Bhandard, S. Ibrahim, O. Adamczyk, M. Porrmann, R. Noé and Y. Achiam, "First real-time data recovery for synchronous QPSK transmission with standard DFB lasers," IEEE Photon. Technol. Lett. 18, 1907-1909 (2006).

[3] A. Leven, N. Kaneda, U.-V. Koc and Y.-K. Chen, "Coherent receivers for practical optical communication systems," in Proceedings of IEEE Conference on Optical Fiber Communications, (Institute of Electrical and Electronics Engineers, Anaheim, 2007), Paper OThK4.

[4] J. Hongo, K. Kasai, M. Yoshida and M. Nakazawa, "1-Gsymbol/s 64-QAM coherent optical transmission over 150 km,” IEEE Photon. Technol. Lett. 19, 638-640 (2007).

[5] M. Nazarathy and E. Simony, "Multichip differential phase encoded optical transmission," IEEE Photon. Technol. Lett. 17, 1133-1135 (2005).

[6] D. Divsalar and M. Simon, "Multiple-symbol differential detection of MPSK," IEEE Trans. Commun. 38, 300-308 (1990).

[7] S. Benedetto and P. Poggiolini, "Theory of polarization shift keying modulation," IEEE Trans. Commun. 40, 708-721 (1992).

[8] E. Ip and J.M. Kahn, "Digital equalization of chromatic dispersion and polarization mode dispersion,” J. Lightwave Technol. 25, 2033-2043 (2007).

[9] S. Tsukamoto, K. Katoh and K. Kikuchi, "Coherent demodulation of optical multilevel phaseshift-keying signals using homodyne detection and digital signal processing," IEEE Photon. Technol. Lett. 18, 1131- 1133 (2006).

[10] C. D. Poole, R. W. Tkach, A. R. Chraplyvy and D. A.Fishman, "Fading in lightwave systems due to polarization-mode dispersion,” IEEE Photon. Technol. Lett. 3, 68-70 (1991).

[11] H. Bülow, W. Baumert, H. Schmuck, F. Mohr, T. Schulz, F. Küppers and W. Weiershausen, "Measurement of the maximum speed of PMD fluctuation in installed field fiber," in Proceedings 
International Journal of Computer Networks \& Communications (IJCNC) Vol.6, No.2, March 2014

of IEEE Conference on Optical Fiber Communications, (Institute of Electrical and Electronics Engineers, San Diego, 1999), Paper OWE4.

[12] H. Sunnerud, C. Xie, M. Karlsson, R. Samuelsson and P. Andrekson, "A comparison between different PMD compensation techniques,” J. Lightwave Technol. 20, 368-378 (2002)..

[13] K.-P. Ho, Phase-Modulated Optical Communication Systems, (Springer, New York, 2005).

[14] K.-P. Ho and J. M. Kahn, "Detection technique to mitigate Kerr effect phase noise," J. Lightwave Technol. 22, 779-783 (2004).

[15] A. P. T. Lau and J. M. Kahn, "Signal design and detection in presence of nonlinear phase noise," J. Lightwave Technol. 25, 3008-3016 (2007).

[16] S.K Mohapatra, R. Bhojray and S.K Mandal, “Analog And Digital Modulation Formats Of Optical Fiber Communication Within And Beyond 100 Gb/S: A Comparative Overview”,IJECET Volume 4, Issue 2, March - April, 2013, ISSN-0976-6472(online).

[17] IEEE Std 802.3ba-2010, Amendment to IEEE Std 802.3-2008: Media Access control parameters, physical layers, and management parameter for $40 \mathrm{~Gb} / \mathrm{s}$ and $100 \mathrm{~Gb} / \mathrm{s}$ operation, June 2010. [18] Gizem, Aksahya \& Ayese, Ozcan (2009) Coomunications \& Networks, Network Books, ABC Publishers.

[18] P.J. Winzer et al., 10 _ 107-Gb/s NRZ-DQPSK transmission at $1.0 \mathrm{~b} / \mathrm{s} / \mathrm{Hz}$ over 12 _ $100 \mathrm{~km}$ including 6 optical routing nodes, in: Proc. OFC 2007, post deadline paper PDP24.

[19] K. Kikuchi, "Coherent detection of phase-shift keying signals using digital carrier-phase estimation," in Proceedings of IEEE Conference on Optical Fiber Communications, (Institute of Electrical and Electronics Engineers, Anaheim, 2006), Paper OTuI4.

[20] P. Serena, A. Orlandini and A. Bononi, "Parametric-Gain approach to the analysis of singlechannel DPSK/DQPSK systems with nonlinear phase noise," J. Lightwave Technol. 24, 2026-2037 (2006).

[21] K.P. Ho and H.C. Wang, "Comparison of nonlinear phase noise and intrachannel four-wave mixing for RZDPSK signals in dispersive transmission systems," IEEE Photon. Technol. Lett. 17, 1426-1428 (2005).

[22] K. Kikuchi, "Phase-diversity homodyne detection of multilevel optical modulation with digital carrier phase estimation,” J. Sel. Top. Quantum Electron. 12, 563-570 (2006).

[23] M. G. Taylor, "Accurate digital phase estimation process for coherent detection using a parallel digital processor," in Proceedings ECOC 2005, Glasgow, UK, 2005, Paper Tu4.2.6.

[24] D.-S. Ly-Gagnon, S. Tsukamoto, K. Katoh and K. Kikuchi, "Coherent detection of optical quadrature phase-shift keying signals with coherent phase estimation,” J. Lightwave Technol. 24, 12-21, (2006).

[25] W. Shieh, X. Yi, and Y. Tang, "Experimental demonstration of transmission of coherent optical OFDM Systems," in Proceedings of IEEE Conference on Optical Fiber Communications, (Institute of Electrical and Electronics Engineers, Anaheim, 2007), Paper OMP2.

[26] W. Shieh and C. Athaudage, "Coherent optical orthogonal frequency division multiplexing," Electron. Lett. 42, 587-589 (2006).

[27] N. Cvijetic, L. Xu and T. Wang, "Adaptive PMD compensation using OFDM in long-haul 10 Gb/s DWDM systems," in Proceedings of IEEE Conference on Optical Fiber Communications, (Institute of Electrical and Electronics Engineers, Anaheim, 2007), Paper OTuA5

[28] A. Lowery and J. Armstrong, "Orthogonal-frequency-division multiplexing for optical dispersion compensation," in Proceedings of IEEE Conference on Optical Fiber Communications, (Institute of Electrical and Electronics Engineers, Anaheim, 2007), Paper OTuA4.

[29] J. Jang, K. B. Lee and Y.-H. Lee, "Transmit power and bit allocations for OFDM systems in a fading channel," in Proceedings of IEEE GLOBECOM, (Institute of Electrical and Electronics Engineers, San Francisco, 2003), pp. 858-862.

[30] D.-S. Ly-Gagnon, "Information recovery using coherent detection and digital signal pocessing for phaseshift- keying modulation formats in optical communication systems," M.S. Thesis, University of Tokyo (2004).

[31] Eugen Lach,Wilfried Idler "modulation formats for $100 \mathrm{G}$ and beyond" ELSEIVIER optical fiber technology 17(2011) 377-386.

[32] J. M. Kahn and K.-P. Ho, "Spectral efficiency limits and modulation/detection techniques for DWDM Systems,", , J. Sel. Top. Quantum Electron. 10, 259-271 (2004).

[33] M. Rohde,C.Caspar,N.Heimes,M.Konitzer,E-J.Bachus and N.Hanik, "Robustness of DPSK direct detection transmission format in standard fiber WDM systems" Electron.Lett,vol 36,pp. 1483-1484,2000. 
International Journal of Computer Networks \& Communications (IJCNC) Vol.6, No.2, March 2014

[34] W. Idler et al., WDM field trial over $764 \mathrm{~km} \mathrm{SSMF}$ with 16 _ $112 \mathrm{~Gb} / \mathrm{s}$ NRZDQPSK copropagating with $10.7 \mathrm{~Gb} / \mathrm{s}$ NRZ, in: ECOC 2010, paper We.8.C.5.

[35] Sano et al., 14-Tb/s (140 _ 111-Gb/s PDM/WDM) CSRZ-DQPSK transmission over 160 $\mathrm{km}$ using 7-THz bandwidth extended L-band EDFAs, in: ECOC 2006, post- Deadline paper Th4.1.1.

[36] W. Idler et al., 16 - 112 Gb/s NRZ-DQPSK WDM transmission over $604 \mathrm{~km}$ SSMF including high PMD fibers, in: OECC 2010, paper 9B1-2.

[37] Michael H. Eiselt et al., Requirements for 100-Gb/s metro networks, in: OFC 2009, paper OTuN6.

[38] Brian Teipen et al., $100 \mathrm{~Gb} / \mathrm{s}$ DPSK-3ASK modulation format for metro networks: experimental results, in: ITG Photonische Netze, 2009.

[39] M. Eiselt, B. Teipen, DPSK-3ASK transmission optimization by adapting modulation Levels, in: APOC 2008, paper 3171-17.

[40] Xiang Liu et al., Transmission of a 448-Gb/s reduced-guard-interval COOFDM signal with a $60-\mathrm{GHz}$ optical bandwidth over $2000 \mathrm{~km}$ of ULAF and five 80-GHz-grid ROADMs, in: OFC 2010, post deadline paper PDPC2.

[41] K. Roberts et al., 100G and beyond with digital coherent signal processing, IEEE Commun. Mag. (2010) 62-69.

[42] Xiang Zhou, Jianjun Yu, Advanced coherent modulation formats and algorithms: higher- order multi-level coding for high-capacity system based on $100 \mathrm{Gbps}$ channel, in: OFC 2010, paper OMJ3.

[43] G. Bosco et al., Performance limits of Nyquist-WDM and CO-OFDM in high speed PM- QPSK systems,IEEE Photon. Technol. Lett. 22 (2010) 1129-1131.

[44] G. Bosco et al., Performance limits of Nyquist-WDM and CO-OFDM in high speed PM- QPSK systems,IEEE Photon. Technol. Lett. 22 (2010) 1129-1131.

[45] Xiang Liu et al., Efficient digital coherent detection of a 1.2-Tb/s 24-carrier no-guard- interval CO-OFDM signal by simultaneously detecting multiple carriers per sampling, in: OFC 2010, paper OWO2.

[46] P. Hofmann, E. E. Basch, S. Gringeri, R. Egorov, D. A. Fishman, and W. A. Thompson, "DWDM Long Haul Network Deployment for the Verizon GNI Nationwide Network," Proc. Optical Fiber Communication Conf. (OFC „,05), Vol. 2, (2005).

[47] M. Nakazawa et al., 256 QAM (64 Gb/s) coherent optical transmission over $160 \mathrm{~km}$ with an optical bandwidth of $5.4 \mathrm{GHz}$, in: OFC 2010, paper OMJ5.

[48] A. Sano et al., 100 _ 120-Gb/s PDM 64-QAM transmission over $160 \mathrm{~km}$ using line widthtolerant pilotless digital coherent detection, in: ECOC 2010, post deadline paper PD2.4.

[49] M. Alfiad et al., Transmission of 11 _ $224 \mathrm{~Gb} / \mathrm{s}$ POLMUX-RZ-16QAM over $1500 \mathrm{~km}$ of LongLine and pure-silica SMF, in: ECOC 2010, paper We.8.C.2.

[50] T. Xia et al., Field experiment with mixed line-rate transmission $(112 \mathrm{~Gb} / \mathrm{s}, 450 \mathrm{~Gb} / \mathrm{s}$, and 1.15 $\mathrm{Tb} / \mathrm{s}$ ) over $3560 \mathrm{~km}$ of installed fiber using filter less coherent receiver and EDFAs only, in: OFC 2011, PDPA3.

[51] Y. More et al., 200-km transmission of 100-Gb/s 32-QAM dual-polarization signals using a digital coherent receiver, in: ECOC 2010, paper 8.4.6.

[52] S. Okamoto et al., 512 QAM (54 Gb/s) coherent optical transmission over $150 \mathrm{~km}$ with an optical bandwidth of $4.1 \mathrm{GHz}$, in: ECOC 2010, post-deadline paper.

[53] M. Rhode, C. Caspar, N. Heimes, M. Konitzer, E. J. Bachus, and N. Hanik, " Robustness of DPSK direct detection transmission format in standard fiber WDM systems," Electronics Letters, 26(17):1483-1484, August 2000.

[54] C. Wree, J. Leibrich, and W. Rosenkranz, "RZ-DQPSK format with high spectral efficiency and high robustness towards fiber nonlinearities," European Conference on Optical Communication (ECOC), 4(9.6.6), September 2002.

[55] Abhijit Banerjee and B. N. Biswas, "Stability Analysis of a Modified PSK Homodyne Optical Receiver", International Journal of Electronics and Communication Engineering \& Technology (IJECET), Volume 3, Issue 2, 2012, pp. 32 - 40, ISSN Print: 0976- 6464, ISSN Online: $0976-6472$.

[56] C.Xie,L.Moller,H.Haunstein and S.Hunsche "comparison of system tolerance to polarization mode dispersion between different modulation formats", IEEE photons. Technol.Lett.vol.15,pp.1168-1170,Aug.2003 
International Journal of Computer Networks \& Communications (IJCNC) Vol.6, No.2, March 2014

[57] $\mathrm{Yu} \mathrm{Yu}$,Bingrong zou,Wenhan $\mathrm{Wu}$ and Xinliang zhang "all optical parallel NRZ- DPSK to RZ-DPSK format conversion at $40 \mathrm{~Gb} / \mathrm{s}$ based on XPM effect in a single SOA".

[58] I. Morita et al., High speed transmission technologies for 100-Gb/s-classEthernet, in: ECOC 2007, invited paper Mo1.3.1.

[59] M. Daikoku et al., 100-Gb/s DQPSK transmission experiment without OTDMfor 100G Ethernet transport, J. Lightw. Technol. 25 (1) (2007).

[60] M. Daikoku, I. Morita, H. Taga, H. Tanaka, T. Kawanishi, T. Sakamoto, T.Miyazaki, T. Fujita, $100 \mathrm{~Gb} / \mathrm{s}$ DQPSK transmission experiment without OTDMfor 100G Ethernet transport, in: OFC 2006, post-deadline paper PDP36.

[61] P.J. Winzer et al., $2000 \mathrm{~km}-\mathrm{WDM}$ transmission of 10 _ $107 \mathrm{~Gb} / \mathrm{s}$ RZ-DQPSK, in:, ECOC 2006, Cannes, post-deadline paper Th4.1.3.

[62] Xiang Zhou, Jianjun Yu, Mei Du, Guodong Zhang, 2 Tb/s (20 _ 107 Gb/s) RZDQPSK straight-line transmission over $1005 \mathrm{~km}$ of standard single mode fiber (SSMF) without Raman amplification in: Proc. OFC 2008, paper OMQ3.

[63] G. Raybon et al., 107-Gb/s transmission over $700 \mathrm{~km}$ and one intermediate ROADMusing Lambda Xtreme_transport system, in: OFC 2008, paper OMQ4.

[64] Mei Du et al., Unrepeatered transmission of $107 \mathrm{~Gb} / \mathrm{s}$ RZ-DQPSK over $300 \mathrm{~km} \mathrm{NZDSF}$ with bi-directional Raman amplification, in: Proc. OFC 2008, paper JThA47.

[65] Brian Teipen, Impact of modulator characteristicson multi-level signal Transmission performance, in: ITG Workshopp Fachgruppe 5.3.1, 2008 (Kiel).

[66] Brian Teipen et al., 107 Gb/s DPSK-3ASK optical transmission over SSMF, in:OFC 2010, paper NMB1.

[67] ITU-T Recommendation G.709, Interfaces for the Optical Transport Network (OTN), December 2009.

[68] M. Eiselt, B.Teipen, DPSK-3ASK transmission optimization by adapting modulation Levels, in: APOC 2008, paper 3171-17.

[69] D. van den Borne et al., Coherent equalization versus direct detection for 111- Gb/s Ethernet transport, in: LEOS Summer Topical Meeting, 2008, paper MA2-4.

[70] J.X. Cai et al., 20Tbit/s capacity transmission over $6860 \mathrm{~km}$, in: Proceedings OFC, 2011, PDPB4.

[71] A.H. Gnauck et al., 10 _ 224-Gb/s WDM transmission of 28-Gbaud PDM 16-QAM on a 50$\mathrm{GHz}$ grid over $1200 \mathrm{~km}$ of fiber, in: OFC 2010, post deadline paper PDPB8.

[72] P.J. Winzer et al., Spectrally efficient long-haul optical networking using 112- Gb/s polarizationmultiplexed 16-QAM, J. Lighw. Technol. 28 (4) (2010).

[73] M. Alfiad et al., Transmission of 11 _ $224 \mathrm{~Gb} / \mathrm{s}$ POLMUX-RZ-16QAM over $1500 \mathrm{~km}$ of LongLine and pure-silica SMF, in: ECOC 2010, paper We.8.C.2.

[74] P.J. Winzer et al., Generation and 1200-km transmission of 448-Gb/s ETDM 56-Gbaud PDM 16-QAM using a single I/Q modulator, in: ECOC 2010, post dead line paper PD2.2.

[75] B.-E. Olsson et al., RF-assisted optical dual-carrier $112 \mathrm{~Gb} / \mathrm{s}$ polarization multiplexed 16QAM transmitter, in: OFC 2010, paper OMK5.

[76] William Shieh, OFDM for adaptive ultra high-speed optical networks, in: OFC2010, paper OWO1.

[77] Fred Buchali et al., Nonlinear limitations in a joint transmission of $100 \mathrm{~Gb} / \mathrm{s}$ OOFDM and 40 Gb/s DPSK over a $50 \mathrm{GHz}$ channel grid, in: OFC 2010, paper OTuL4.

[78] S. Chandrasekhar et al., Transmission of a 1.2-Tb/s 24-carrier no-guard interval coherent OFDM super channel over 7200-km of ultra-large-area fiber, in: ECOC 2009, post-deadline paper PD 2.6.

[79] Haiying Julie Zhu, Roshdy H.M. Hafez and John Sydor, "Cross Layer Scheduling Algorithms for Different Rate Traffic OFDM Broadband Wireless Systems”, IJCNC Vol No.1,No.3,October 2009

[80] Asim M. Mazin and Garth V. Crosby, "Reducing the Peak Average Power Ratio of MIMO-OFDM Systems", IJCNC Vol.5, No.3, May 2013.

\section{Authors}

Sumant Ku Mohapatra was born in Bhubaneswar, Orissa, India . He received the B.Tech degree from ITER, Bhubaneswar, India in 2002 \& M.Tech(Electronics \& Instrumentation Engg) from GIET, BPUT, Odisha in 2011.. His research interest are in signal and image processing, optical fiber communication and wireless communication. Currently he is

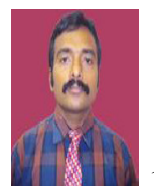


working in Trident Academy of Technology, B.P.U.T, Bhubaneswar, Odisha, India as an Assistant Professor in the Department of

Ramya Ranjan Choudhury was born in the Balasore district, Orissa, India . He received the B.Tech degree from Silicon Institute of Technology, Bhubaneswar, India in 2008 \& M.Tech (Electronics \& Communication Engg) from C.E.B, BPUT, Odisha in 2012. His research interests include digital signal processing, digital image processing, optical fiber communication and wireless communication. He is currently working in Trident Academy of Technology, Bhubaneswar, Odisha, India as an Assistant Professor.

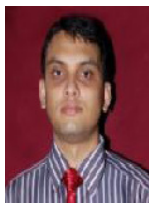

Rabindra Bhojray was born in the Cuttack district, Odisha, India . He received the graduate Engineering from I.E.T.E New Delhi, India in 1998 and received the master engineering in field of communication system engineering from U.C.E, Burla, Orissa (At present V.S.S.U.T, Burla), in 2001 and Ph.D. (continuing). His research interest are in signal and image processing ,optical fiber communication and wireless communication.

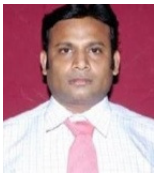
Currently he is an associate professor in Trident Academy of Technology, B.P.U.T, Bhubaneswar, Odisha.

Pravanjan Das was born in the Cuttack district, Odisha, India . He received the B.Tech degree from TAT, Bhubaneswar, India in 2010 \& M.Tech(Communication Systems) from SVNIT,Surat,Gujrat in 2013. Currently he is working in Trident Academy of Technology, B.P.U.T, Bhubaneswar, Odisha, as an Assistant Professor. His research interest are in signal processing, and wireless communication.

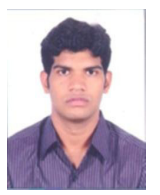

